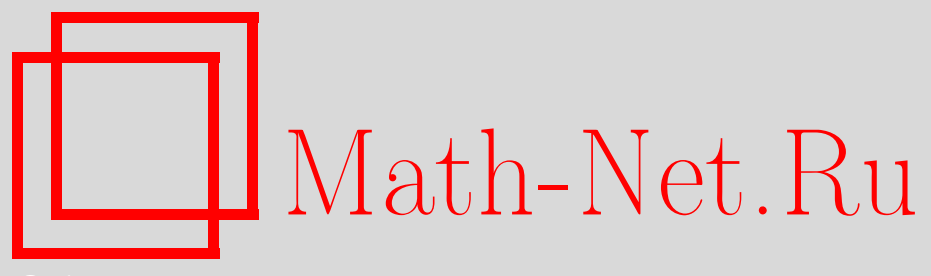

А. Ю. Зайцев, Точность сильной гауссовской аппроксимации для сумм независимых случайных векторов, УМН, 2013, том 68, выпуск 4, 129-172

DOI: https://doi.org/10.4213/rm9537

Использование Общероссийского математического портала Math-Net.Ru подразумевает, что вы прочитали и согласны с пользовательским соглашением http://www.mathnet.ru/rus/agreement

Параметры загрузки:

IP: 34.227 .88 .159

26 апреля 2023 г., 11:34:05

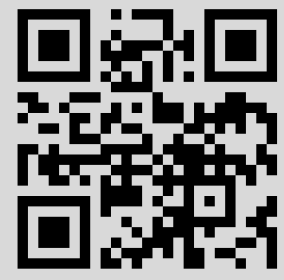




\title{
Точность сильной гауссовской аппроксимации для сумм независимых случайных векторов
}

\author{
А. Ю. Зайцев \\ В статье дан обзор недавних результатов о точности сильной гауссов- \\ ской аппроксимации для сумм независимых случайных векторов. В них \\ содержатся многомерные обобщения одномерных результатов Я. Комло- \\ ша, П. Майора и Г. Тушнади, а также А. И. Саханенко и усиления много- \\ мерных результатов У. Айнмаля. Даны также бесконечномерные аналоги \\ этих результатов. \\ Библиография: 102 названия.
}

Ключевые слова: многомерный принцип инвариантности, сильная аппроксимация, суммы независимых случайных векторов.

DOI: $10.4213 / \mathrm{rm} 9537$

\section{СоДЕРЖАНИЕ}

1. Введение ............................................

2. Точность аппроксимации в задаче (В) для векторов с конечными экспоненциальными моментами ........................... 134

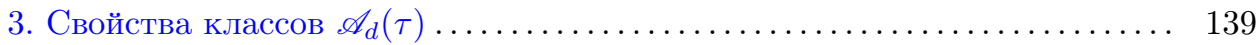

4. Точность аппроксимации в принципе инвариантности Штрассена...... 143

5. Точность аппроксимации в задаче (В) для сумм неодинаково распределенных векторов с конечными моментами $\mathrm{E} H\left(\left\|X_{j}\right\|\right)<\infty \ldots \ldots, 146$

6. Точность аппроксимации в задаче (В) для сумм одинаково распределенных векторов с конечными моментами $\mathrm{E} H(\|X\|)<\infty \ldots \ldots \ldots .150$

7. Точность аппроксимации в задаче (B) для сумм неодинаково распределенных векторов с конечными степенными моментами ....................

8. Точность аппроксимации в задаче (В) для сумм одинаково распределенных векторов с конечными степенными моментами ........... 156

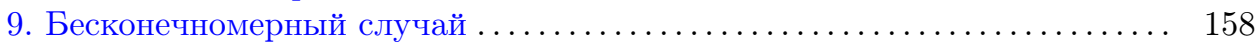

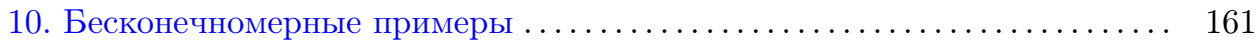

11. Правильные по порядку оценки в бесконечномерном случае ........ 163

Список литературы ..................................... 166

Работа выполнена при поддержке РФФИ (грант № 13-01-00256), программы "Ведущие научные школы" (грант НШ-1216.2012.01) и программы фундаментальных исследований РАН "Современные проблемы теоретической математики".

(C) А. Ю. ЗАйцев, 2013 


\section{1. Введение}

В статье дан обзор недавних результатов о точности сильной гауссовской аппроксимации для сумм независимых случайных векторов.

Пусть $\xi_{1}, \xi_{2}, \ldots$ - независимые $\mathbb{R}^{d}$-значные случайные векторы с нулевыми средними и $\mathbb{D}_{n}=\operatorname{cov} S_{n}$ - ковариационные операторы сумм $S_{n}=\sum_{i=1}^{n} \xi_{i}$. Согласно центральной предельной теореме, при некоторых простых моментных условиях распределения нормированных сумм $\mathbb{D}_{n}^{-1 / 2} S_{n}$ близки к стандартному гауссовскому распределению. Принцип инвариантности является естественным обобщением центральной предельной теоремы. Он утверждает, что в некотором смысле распределение всей последовательности $\mathbb{D}_{n}^{-1 / 2} S_{1}, \ldots, \mathbb{D}_{n}^{-1 / 2} S_{n}$ близко к распределению последовательности $\mathbb{D}_{n}^{-1 / 2} T_{1}, \ldots, \mathbb{D}_{n}^{-1 / 2} T_{n}$, где $T_{n}=\sum_{i=1}^{n} Y_{i}$ и $Y_{1}, Y_{2}, \ldots$ - соответствующая последовательность независимых гауссовских векторов (это означает, что $Y_{i}$ имеют те же средние и те же ковариационные операторы, что и $\left.\xi_{i}, i=1,2, \ldots\right)$.

Задача об оценивании точности сильной аппроксимации намного содержательнее, чем задача об оценивании близости распределений. Последовательности с близкими распределениями требуется построить на одном вероятностном пространстве так, чтобы они оказались близки между собой. Эта задача является одной из самых важных в области предельных теорем теории вероятностей, поскольку многие известные результаты могут быть получены как следствия утверждений о сильной аппроксимации последовательностей сумм соответствующими гауссовскими последовательностями. Это относится к функциональному закону повторного логарифма Штрассена, к теоремам об аппроксимации распределений функционалов от нарастающих сумм независимых слагаемых распределениями функционалов от броуновского движения, к теоремам о больших уклонениях, а также к оценкам скорости сходимости в принципе инвариантности в метрике Прохорова (см., например, [10], [12], [19], [21]).

Оценивание точности сильной аппроксимации сумм независимых случайных векторов в принципе инвариантности имеет огромное количество возможных применений во всех областях науки, в которых исследователь имеет дело со случайными блужданиями. Это и физика, и биология, и экономика, и финансовая математика. Оценки позволяют исследовать свойства различных случайных блужданий на основе известных и хорошо изученных свойств одного процесса - многомерного броуновского движения.

Принцип инвариантности стал результатом длительного развития теории вероятностей. В классической работе П. Эрдёша и М. Каца [36] было замечено, что предельные распределения некоторых функционалов от нарастающих сумм независимых одинаково распределенных случайных величин с конечной дисперсией не зависят от распределения слагаемых и, следовательно, могут быть вычислены в случае, когда распределение слагаемых имеет конкретный простой вид, удобный для проведения вычислений. Идеи Эрдёша и Каца были 
развиты Дж. Дубом [26], М. Донскером [24], [25], Ю. В. Прохоровым [62] и другими, что привело к созданию теории слабой сходимости вероятностных мер на метрических пространствах (см., например, монографию П. Биллингсли [7]).

Принцип инвариантности Донскера-Прохорова говорит о том, что распределения должным образом центрированных и нормированных случайных ломаных, построенных по нарастающим суммам независимых случайных величин с конечными дисперсиями, при определенных условиях слабо сходятся к распределению стандартного броуновского движения. Отсюда следует и слабая сходимость распределений ограниченных непрерывных функционалов от ломаных к распределениям тех же функционалов от броуновского движения.

Введем обозначения, которые будут использоваться ниже. Распределение случайного вектора $\xi$ будет обозначаться $\mathscr{L}(\xi)$. Соответствующий ковариационный оператор будет обозначаться $\operatorname{cov} \xi$ или $\operatorname{cov} V$, если $V=\mathscr{L}(\xi)$. В дальнейшем $\log ^{*} b=\max \{1, \log b\}$ при $b>0$. Здесь $\log$ используется для обозначения натурального логарифма. Мы будем обозначать символом $c$ положительные абсолютные постоянные, которые могут быть различными в различных местах текста. Кроме того, символ $a(\cdot, \ldots, \cdot)$ будет обозначать положительные величины, зависящие только от своих аргументов, причем не обязательно одни и те же. В дальнейшем $\mathbb{I}_{d}$ - тождественный оператор в $\mathbb{R}^{d}$, а $\|\cdot\|$ и $\langle\cdot, \cdot\rangle-$ евклидова норма и скалярное произведение.

Точность сильной гауссовской аппроксимации для сумм независимых случайных векторов обычно оценивается в двух следующих различных, но тесно связанных ситуациях. Оценивание точности сильной аппроксимации в принципе инвариантности может быть сведено к этим задачам.

(A) Требуется построить на одном вероятностном пространстве последовательность независимых одинаково распределенных случайных векторов $X_{1}$, $X_{2}, \ldots$ (с заданным распределением $\mathscr{L}(X)$ с нулевым средним и $\mathrm{E}\|X\|^{2}<\infty$ ) и последовательность независимых гауссовских случайных векторов $Y_{1}, Y_{2}, \ldots$ таким образом, чтобы

$$
\mathscr{L}\left(X_{j}\right)=\mathscr{L}(X), \quad \mathrm{E} Y_{j}=0, \quad \operatorname{cov} Y_{j}=\operatorname{cov} X, \quad j=1,2, \ldots,
$$

и чтобы

$$
\left\|\sum_{j=1}^{n} X_{j}-\sum_{j=1}^{n} Y_{j}\right\|=O(f(n)) \quad \text { или } \quad o(f(n)) \quad \text { почти наверное (п.н.) }
$$

при $n \rightarrow \infty$ для последовательности $f(n)$, стремящейся к бесконечности как можно медленнее.

(В) Пусть $\xi_{1}, \ldots, \xi_{n}$ - независимые (вообще говоря, неодинаково распределенные) случайные векторы с нулевыми математическими ожиданиями и конечными моментами второго порядка. Требуется построить на одном вероятностном пространстве независимые случайные векторы $X_{1}, \ldots, X_{n}$ и независимые гауссовские случайные векторы $Y_{1}, \ldots, Y_{n}$ таким образом, чтобы

$$
\mathscr{L}\left(X_{j}\right)=\mathscr{L}\left(\xi_{j}\right), \quad \mathrm{E} Y_{j}=0, \quad \operatorname{cov} Y_{j}=\operatorname{cov} X_{j}, \quad j=1, \ldots, n,
$$

и чтобы величина

$$
\Delta_{n}(X, Y)=\max _{1 \leqslant s \leqslant n}\left\|\sum_{j=1}^{s} X_{j}-\sum_{j=1}^{s} Y_{j}\right\|
$$


была по возможности мала с достаточно большой вероятностью.

Следует обратить внимание на то, что задачи сформулированы для ненормированных последовательностей сумм. Их сближение можно заметить только после подходящей перенормировки.

Наиболее важными являются результаты, дающие решение задачи (В). Как правило, именно они вносят решающий вклад при решении задачи (А). Ниже в этом разделе мы будем говорить в основном о задаче (B). Задаче $(\mathrm{A})$ посвящен отдельный раздел 4. Подчеркнем, что задачу (A) мы рассматриваем только для одинаково распределенных слагаемых.

Для краткости мы будем вместо выписывания перечисленных выше свойств векторов $X_{1}, \ldots, X_{n}$ и $Y_{1}, \ldots, Y_{n}$ просто говорить, что существует построение, обладающее дополнительными свойствами, явно указываемыми в тексте. Для упрощения обозначений мы будем отождествлять векторы $\xi_{1}, \ldots, \xi_{n}$ и векторы $X_{1}, \ldots, X_{n}$, формулируя условия как условия на распределения векторов $X_{1}, \ldots, X_{n}$.

Если векторы $X_{1}, \ldots, X_{n}$ одинаково распределены, в конечномерном или бесконечномерном пространстве, в формулировках результатов иногда будет участвовать случайный вектор $X$ с $\mathscr{L}(X)=\mathscr{L}\left(X_{1}\right)$. Независимые копии $X_{1}, \ldots, X_{n}$ вектора $X$ требуется построить на одном вероятностном пространстве с последовательностью независимых гауссовских случайных векторов $Y_{1}, \ldots, Y_{n}$.

Точность сильной аппроксимации в одномерном принципе инвариантности изучалась многими авторами (см., например, работы Ю.В. Прохорова [62], А. В. Скорохода [82], Ф. Штрассена [84], [86], А.А. Боровкова [9], М. Чёрге и П. Ревеса [20], Я. Комлоша, П. Майора и Г. Тушнади [50], У. Айнмаля [28], У. Айнмаля и Д. Мейсона [34], А. И. Саханенко [72], [73], [75], [77], [78], С. Чаттерджи [17] и библиографию в книге М. Чёрге и П. Ревеса [21] и в статьях Ш. Чёрге и П. Холла [19], П. Майора [55], А. А. Боровкова и А. И. Саханенко [11], [12], Ки-ман Шао [63] и автора [98]). История вопроса до середины девяностых годов прошлого века изложена в обзорной статье А. А. Боровкова, А. А. Могульского и А. И. Саханенко [10].

В формулировках результатов часто участвуют не последовательности нарастающих сумм, а случайные ломаные и траектории броуновского движения. Но это не приводит к существенному изменению вида аппроксимационных неравенств. Для обоснования соответствующих оценок достаточно использовать стандартное средство доказательства теорем о сильной аппроксимации лемму А из работы И. Беркеша и У. Филиппа [4], а также так называемые максимальные неравенства.

Первые оценки скорости сходимости в принципе инвариантности появились в статье Ю.В. Прохорова [62]. В ней впервые систематически исследовались предельные теоремы для случайных процессов, было определено и оценивалось расстояние, получившее в дальнейшем имя автора работы [62]. Ю. В. Прохоров первым использовал построение независимых случайных величин на одном вероятностном пространстве для получения оценок в принципе инвариантности. 
Его метод состоял в использовании так называемых квантильных преобразований, при которых суммы по блокам случайных величин $X_{j}$ определяются как неубывающие функции от соответствующих независимых сумм случайных величин $Y_{j}$.

А. В. Скороход [82] разработал метод построения близких последовательностей сумм независимых одинаково распределенных случайных величин, задавая их на одном вероятностном пространстве с подходящим образом выбранным винеровским процессом. Довольно долго именно этот метод позволял получать наилучшие оценки. Он известен теперь как метод вложения Скорохода. Оказалось, однако, что возможности метода ограничены. Он давал оценки с оптимальной точностью аппроксимации только при предположении о существовании моментов невысоких порядков. Но и получаемые при этом оценки давали относительно невысокую скорость сходимости. Более сильные моментные предположения позднее позволили получить намного более сильные оценки с помощью других методов (см. [20], [50]). Вложение Скорохода до сих пор успешно используется при сильной аппроксимации сумм зависимых случайных величин, хотя оптимальные по порядку оценки, соответствующие независимому случаю, были получены лишь недавно за счет сведения случая зависимых величин к независимым (см. [5], [6]). Используется вложение Скорохода и при совместной сильной аппроксимации сумм и соответствующих времен пребывания в принципе инвариантности для локальных времен (см. [14]).

Я. Комлош, П. Майор и Г. Тушнади (KMT) [50] предложили новый метод диадической аппроксимации. С его помощью они получили оптимальные оценки точности сильной гауссовской аппроксимации последовательностей сумм независимых одинаково распределенных случайных величин. При этом существенно использовалась одинаковая распределенность слагаемых и не выписывалась в явном виде зависимость констант от их распределения.

В работе KMT [50] содержится также результат о сильной гауссовской аппроксимации одномерного эмпирического процесса. В настоящей статье мы не затрагиваем вопрос о сильной аппроксимации эмпирических процессов. Упомянем только то, что эмпирические процессы и процессы нарастающих сумм тесно связаны между собой, так что из результатов для одних процессов иногда удается вывести содержательные результаты для других (см., например, [8], [18], [67]). Существуют также результаты, имеющие отношение к обеим задачам, когда доказываются оценки для сильной аппроксимации взвешенных сумм одинаково распределенных слагаемых, равномерные по достаточно широким классам весов (см., например, [45], [49], [57], [65], [66]).

Упомянем недавнюю работу С. Чаттерджи [17], в которой результат KMT [50] для простейшего случая распределений из симметричной схемы Бернулли был получен с использованием другой техники оценивания погрешностей в методе диадической аппроксимации КМТ. Основными элементами этой техники являются теорема о неподвижной точке и известный метод Чена-Стейна.

Дальнейший прогресс для процессов нарастающих сумм в одномерном случае был достигнут в основном в работах А. И. Саханенко [72], [73], [75], [77], [78]. В работе [72] он обобщил и существенно уточнил результаты KMT в случае 
неодинаково распределенных $\mathbb{R}^{1}$-значных случайных величин с конечными экспоненциальными моментами (см. теорему 1). Ему удалось свести зависимость констант от неодинаковых распределений слагаемых к зависимости от единого параметра, характеризующего выполнение для распределений слагаемых условия, эквивалентного условиям известного неравенства Бернштейна (см. [60]). Затем, в работе [73], А. И. Саханенко использовал результат [72] для получения оптимальных оценок точности сильной гауссовской аппроксимации сумм независимых случайных величин $X_{j}$ с конечными моментами вида $\mathrm{E} H\left(\left|X_{j}\right|\right)$, где $H(x)$ - монотонная функция, растущая не медленнее, чем $x^{2}$, и не быстpeе, чем $e^{c x}$. Наиболее полные и изящно сформулированные результаты были получены в случае $H(x)=x^{\gamma}, \gamma \geqslant 2$ (см. теорему 6). В работе [77] А. И. Саханенко уточнил результаты [73], сформулировав оценки в терминах урезанных моментов. В недавней работе [78] ему удалось получить результат, из которого следуют все результаты KMT [50] для сумм независимых случайных величин, относящиеся как к задаче (В), так и к задаче (А).

\section{2. Точность аппроксимации в задаче (В) для векторов с конечными экспоненциальными моментами}

В этом разделе мы остановимся на наиболее важном случае, когда слагаемые имеют конечные экспоненциальные моменты. А. И. Саханенко [72] обобщил и существенно уточнил результаты КМТ в случае неодинаково распределенных случайных величин. Он рассмотрел следующие классы одномерных распределений:

$$
\mathscr{S}_{1}(\tau)=\left\{\mathscr{L}(\xi): \mathrm{E} \xi=0, \mathrm{E}|\xi|^{3} \exp \left(\tau^{-1}|\xi|\right) \leqslant \tau \mathrm{E}|\xi|^{2}\right\}, \quad \tau>0 .
$$

В препринте автора [88] было замечено, что классы $\mathscr{S}_{1}(\tau)$ эквивалентны классам распределений $\mathscr{B}_{1}(\tau)$, удовлетворяющих условиям неравенства Бернштейна (см. определение (17) ниже), в том смысле, что если одно из условий принадлежности к классу выполняется с параметром $\tau$, то второе справедливо с параметром $c \tau$, где $c$ - некоторая положительная абсолютная константа.

Основной результат [72] для сумм независимых случайных величин с распределениями из класса $\mathscr{S}_{1}(\tau)$ формулируется следующим образом.

Теорема 1. Предположим, что $\tau>0$ u $X_{1}, \ldots, X_{n}$ - независимые случайные величины с $\mathscr{L}\left(X_{j}\right) \in \mathscr{S}_{1}(\tau), j=1, \ldots, n$. Тогда существует такое построение, что

$$
\mathrm{E} \exp \left(\frac{c \Delta(X, Y)}{\tau}\right) \leqslant 1+\frac{B}{\tau},
$$

где с-абсолютная константа и $B^{2}=\mathrm{E} X_{1}^{2}+\cdots+\mathrm{E} X_{n}^{2}$.

В работе [50] предполагалось, что $X, X_{1}, \ldots, X_{n}$ одинаково распределены и $\mathrm{E} e^{h X}<\infty$ при $h \in V$, где $V \subset \mathbb{R}^{1}-$ некоторая окрестность нуля. Результат KMT [50] следует из теоремы 1 . Легко видеть, что существует такое $\tau(F)>0$, 
что $F=\mathscr{L}(X) \in \mathscr{S}_{1}(\tau(F))$. Применяя неравенство Чебышёва, получим, что из (4) следует, что

$$
\mathrm{P}\left\{\frac{c \Delta(X, Y)}{\tau(F)} \geqslant x\right\} \leqslant \exp \left(\log \left(1+\frac{\sqrt{n \mathrm{E} X^{2}}}{\tau(F)}\right)-x\right), \quad x>0
$$

Неравенство (5) содержит больше информации, чем первоначальная формулировка KMT, в которой константы зависят от $F$ неявным образом. В (5) зависимость констант от распределения $F$ выписана в явном виде. Величину $\tau(F)$ можно легко вычислить или оценить для любого конкретного распределения $F$. Более того, в теореме 1 случайные величины, вообще говоря, неодинаково распределены.

Первые попытки получить оценки в многомерном случае (см. работы В. В. Городецкого [44], И. Беркеша и У. Филиппа [4], У. Филиппа [61], А. А. Боровкова и А. И. Саханенко [11], Э. Бергера [3], У. Айнмаля [28]-[30], А. И. Саханенко [76]) имели лишь частичный успех. Большинство из упомянутых работ содержат результаты для случая $H(x)=x^{\gamma}$ при достаточно малых значениях $2 \leqslant \gamma \leqslant 4$ или оценки расстояния Прохорова и ему подобных величин. Оценивание расстояния Прохорова (вместе с теоремой Штрассена-Дадли) дает возможность получать утверждения о сильной аппроксимации для конкретных значений аргумента $z$ (см. теорему 8). Это означает, что вероятностное пространство зависит от этого $z$.

В 1989 г. была опубликована работа У. Айнмаля [31], в которой содержались многомерные аналоги результатов КMT, близкие к оптимальным. Для оценивания зависимости констант от распределения слагаемых он использовал класс распределений $\mathscr{S}_{d}(\tau)$, определяемый так же, как $\mathscr{S}_{1}(\tau)$, с заменой модуля на евклидову норму. Айнмаль [31] получил оптимальные результаты для сумм независимых одинаково распределенных случайных векторов с $\mathrm{E} H(\|X\|)<\infty$ для некоторых функций $H$ с вероятностным пространством, зависящим от $z$. В частности, он получил оценки с оптимальной точностью в случае $H(x)=x^{\gamma}$, $\gamma>3$. Случай $2<\gamma \leqslant 3$ был исследован ранее Бергером [3] (см. также работу А. И. Саханенко [76]). Однако в общем случае векторов с конечными экспоненциальными моментами результаты Айнмаля [31] не давали оптимальную точность аппроксимации.

Автор [93]-[95] избавился от излишнего логарифмического множителя в результате Айнмаля [31] и получил многомерный аналог результата KMT [50] для векторов с конечными экспоненциальными моментами (см. теорему 2). В теореме 2 случайные векторы, вообще говоря, неодинаково распределены. Впрочем, они имеют один и тот же единичный ковариационный оператор $\mathbb{I}_{d}$. Поэтому вопрос о получении адекватного многомерного обобщения основных результатов Саханенко [72] оставался открытым. Для векторов с конечными экспоненциальными моментами это обобщение приводится ниже в теореме 3.

Пусть $\mathscr{A}_{d}(\tau), \tau \geqslant 0, d \in \mathbb{N},-$ класс $d$-мерных распределений, введенный в работе автора [89]. Класс $\mathscr{A}_{d}(\tau)$ (с фиксированным $\left.\tau \geqslant 0\right)$ состоит из $d$-мерных 
распределений $F$, для которых функция

$$
\varphi(z)=\varphi(F, z)=\log \int_{\mathbb{R}^{d}} e^{\langle z, x\rangle} F\{d x\} \quad(\varphi(0)=0)
$$

определена и аналитична при $\|z\| \tau<1, z \in \mathbb{C}^{d}$ и

$$
\left|d_{u} d_{v}^{2} \varphi(z)\right| \leqslant\|u\| \tau\langle\mathbb{D} v, v\rangle
$$

для всех $u, v \in \mathbb{R}^{d}$ и $\|z\| \tau<1$, где $\mathbb{D}=\operatorname{cov} F$, а $d_{u} \varphi-$ производная функции $\varphi$ в направлении $u$.

Tеорема 2 [93], [95]. Пусть $\tau \geqslant 1, \alpha>0 u X_{1}, \ldots, X_{n}$ - независимые $d$-мерные случайные векторы с $\mathscr{L}\left(X_{k}\right) \in \mathscr{A}_{d}(\tau), \mathrm{E} X_{k}=0, \operatorname{cov} X_{k}=\mathbb{I}_{d}, k=$ $1, \ldots, n$. Тогда существует такое построение, что

$$
\mathrm{E} \exp \left(\frac{a_{1} \Delta_{n}(X, Y)}{\tau d^{7 / 2} \log ^{*} d}\right) \leqslant \exp \left(a_{2} d^{9 / 4+\alpha} \log ^{*} \frac{n}{\tau^{2}}\right)
$$

где $a_{1}, a_{2}$ - положительные величины, зависящие только от $\alpha$.

СледствиЕ 1. В условиях теоремы 2 для всех $x \geqslant 0$ справедливо следующее неравенство:

$$
\mathrm{P}\left\{\Delta_{n}(X, Y) \geqslant \frac{a_{2} \tau d^{23 / 4+\alpha} \log ^{*} d \log ^{*}\left(n / \tau^{2}\right)}{c_{1}(\alpha)}+x\right\} \leqslant \exp \left(-\frac{a_{1} x}{\tau d^{7 / 2} \log ^{*} d}\right) .
$$

Легко видеть, что если $V \subset \mathbb{R}^{d}$ - некоторая окрестность нуля и для некоторого $d$-мерного случайного вектора $\xi$ выполняется $\mathrm{E} e^{\langle h, \xi\rangle}<\infty$ при всех $h \in V$, то $F=\mathscr{L}(\xi) \in \mathscr{A}_{d}(c(F))$. В разделе 3 мы приведем некоторые простые и полезные свойства классов $\mathscr{A}_{d}(\tau)$, которые играют важную роль в доказательстве теоремы 2. Теорема 2 влечет в одномерном случае как теорему 1 для одинаково распределенных случайных величин с конечными экспоненциальными моментами, так и результат KMT [50].

В [95] теорема 2 была сформулирована и доказана при фиксированном $n$. Это означает, что вероятностное пространство зависело от этого $n$. Однако применение результата при фиксированном $n$ позволяет получить такое построение, что (8) выполняется при всех $n$ одновременно на одном и том же вероятностном пространстве. Достаточно взять независимые построения из формулировки теоремы 2 с фиксированным $n=2^{2^{m}}, m=1,2, \ldots$, как было сделано в одномерном случае в работе КМТ [50]. Это построение подробно описано в работе автора [97], в которой содержится также доказательство следующего утверждения, вытекающего из теоремы 2.

СлЕДСТвиЕ 2. Предположим, что случайный вектор $X$ имеет конечные экспоненциальные моменты $\mathrm{E} e^{\langle h, X\rangle}$ nри $h \in V$, где $V \subset \mathbb{R}^{d}$ - некоторая окрестность нуля, $u \mathrm{E} X=0$. Тогда существует такое построение с одинаково распределенными $X_{k}$ с $\mathscr{L}\left(X_{k}\right)=\mathscr{L}(X), k=1,2, \ldots$, ито

$$
\sum_{k=1}^{n} X_{k}-\sum_{k=1}^{n} Y_{k}=O(\log n) \quad \text { п.н. }
$$


Аналог этого результата был получен Айнмалем [31] при дополнительных ограничениях на гладкость распределений $\mathscr{L}(X)$. Айнмаль доказал также аналог теоремы 2 (см. [31; теорема 10]), но только для достаточно гладких распределений $\mathscr{L}(X)$. В одномерном случае утверждение следствия 2 было доказано в работе KMT [50]. В [50] было также отмечено, что из результатов П. Бартфаи [1] следует, что точность аппроксимации в (10) наилучшая из возможных для негауссовских векторов $X$.

Следующее утверждение является уточнением следствия 2.

СлеДСТвИЕ 3 [97]. Предположим, что распределение случайного вектора $X$ таково, что $\mathscr{L}\left(\mathbb{D}^{-1 / 2} X\right) \in \mathscr{A}_{d}(\tau)$, где $\tau \geqslant 1$, a $\mathbb{D}=\operatorname{cov} \mathscr{L}(X)$ - обратимыи оператор. Пусть $\sigma^{2}, \sigma>0$, - максимальное собственное число оператора $\mathbb{D}$. Тогда для любого $\alpha>0$ существует такое построение с одинаково распределенными $X_{k}$ с $\mathscr{L}\left(X_{k}\right)=\mathscr{L}(X), k=1,2, \ldots$, что

$$
\mathrm{P}\left\{\limsup _{n \rightarrow \infty} \frac{1}{\log n}\left\|\sum_{k=1}^{n} X_{k}-\sum_{k=1}^{n} Y_{k}\right\| \leqslant a_{3} \sigma \tau d^{23 / 4+\alpha} \log ^{*} d\right\}=1,
$$

где $a_{3}$ зависит только от $\alpha$.

В теореме 2 и следствии 3 присутствует условие $\tau \geqslant 1$. Случай малых $\tau$ был исследован в работе [40]. В этой работе показано, что при дополнительных ограничениях на гладкость распределения $\mathscr{L}(X)$ выражение в правой части неравенства в формуле (11) может быть сколь угодно малым, если параметр $\tau$ достаточно мал. Ясно, что утверждения теоремы 2 и следствия 3 становятся сильнее при малых $\tau$. В работе [40] можно найти простые примеры, для которых достаточно громоздкие условия гладкости выполнены. Точность аппроксимации оказывается лучше, когда слагаемые имеют гладкие распределения, близкие к гауссовским (см. неравенства (15) и (16) в разделе 3 ).

Пусть $X_{1}, \ldots, X_{n}$ - независимые $d$-мерные случайные векторы и мы занимаемся решением задачи (B). В теоремах $3,7,10$ и следствиях 5 и 6 будут использоваться варианты следующего условия.

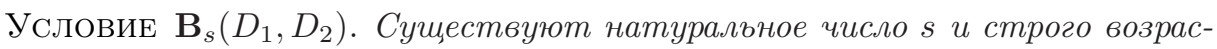
тающая последовательность неотрицательных целых чисел $m_{0}=0, m_{1}, \ldots$, $m_{s}=n$, удовлетворяющие следуюшим условиям. Пусть

$$
\zeta_{k}=X_{m_{k-1}+1}+\cdots+X_{m_{k}}, \quad \operatorname{cov} \zeta_{k}=\mathbb{B}_{k}, \quad k=1, \ldots, s .
$$

Тогда для всех $v \in \mathbb{R}^{d}$

$$
D_{1}\|v\|^{2} \leqslant\left\langle\mathbb{B}_{k} v, v\right\rangle \leqslant D_{2}\|v\|^{2}, \quad k=1, \ldots, s,
$$

с некоторыми положительными величинами $D_{1}$ u $D_{2}$.

Смысл условия $\mathbf{B}_{s}\left(D_{1}, D_{2}\right)$ состоит в том, что сумма $X_{1}+\cdots+X_{n}$ разбивается на $s$ сумм по блокам слагаемых, у которых все собственные числа ковариационных операторов находятся между $D_{1}$ и $D_{2}$. При этом отношения $D_{2} / D_{1}$ 
в упомянутых выше результатах считаются константами, от которых могут зависеть постоянные $a_{j}$, участвующие в оценках. Так что условие $\mathbf{B}_{s}\left(D_{1}, D_{2}\right)$ можно рассматривать как условие равномерной невырожденности ковариационных операторов сумм по блокам слагаемых.

Впервые идея налагать ограничения на суммы по блокам случайных величин использовалась А. И. Саханенко в теоремах 8 и 10 в работе [72].

В работе [96] автор обобщил теорему 2 на случай неодинаково распределенных слагаемых с различными ковариационными операторами и доказал следующую теорему.

Теорема 3. Пусть $\tau \geqslant 1, \alpha>0 u X_{1}, \ldots, X_{n}$ - независимые $d$-мерные случайные векторы с $\mathrm{E} X_{j}=0, j=1, \ldots, n$. Предположим, что выполнено условие $\mathbf{B}_{s}\left(C_{1}, C_{2}\right)$ с некоторыми положительными постоянными $C_{1}$ и $C_{2}$, причем суммы по блокам слагаемых (12) таковы, что

$$
\mathscr{L}\left(\zeta_{k}\right) \in \mathscr{A}_{d}(\tau), \quad k=1, \ldots, s
$$

Тогда существует такое построение, что

$$
\mathrm{E} \exp \left(\frac{a_{4} \Delta_{n}(X, Y)}{\tau d^{9 / 2} \log ^{*} d}\right) \leqslant \exp \left(a_{5} d^{3+\alpha} \log ^{*} \frac{s}{\tau^{2}}\right),
$$

где $a_{4}, a_{5}$ - положительные величины, зависящие только от $\alpha, C_{1} u C_{2}$.

Теорема 3 дает адекватное многомерное обобщение теоремы 1. В теореме 3 случайные векторы, вообще говоря, неодинаково распределены. Впрочем, они должны удовлетворять условию $\mathbf{B}_{s}\left(C_{1}, C_{2}\right)$ на ковариационные операторы. Это условие порождает условия $\mathbf{B}_{s}(\cdot, \cdot)$ в теоремах 7,10 и следствиях 5 и 6 . Правдоподобная гипотеза состоит в том, что условие (13) в формулировке теоремы 3 может быть ослаблено. То же самое можно сказать об условиях теорем 7, 10 и следствий 5 и 6 . По крайней мере, подобных ограничений не появляется в условиях неравенства (15) (которое, впрочем, не имеет прямого отношения к принципу инвариантности). Но для доказательства этой гипотезы могут потребоваться некоторые новые идеи и методы.

Если параметр $\tau \geqslant 1$ в теореме 3 существенно больше единицы, то применять теорему 3 следует к нормированным случайным векторам $X_{j} / \tau$. Тогда

$$
\mathscr{L}\left(\frac{\zeta_{k}}{\tau}\right) \in \mathscr{A}_{d}(1), \quad k=1, \ldots, s,
$$

и для справедливости утверждения теоремы 3 достаточно вместо $\mathbf{B}_{s}\left(C_{1}, C_{2}\right)$ предположить, что выполнено условие $\mathbf{B}_{s}\left(C_{1} \tau^{2}, C_{2} \tau^{2}\right)$. Ясно, что это условие налагает меньшие ограничения на распределения случайных векторов $X_{j}$ по сравнению с $\mathbf{B}_{s}\left(C_{1}, C_{2}\right)$. При этом $\log ^{*}\left(s / \tau^{2}\right)$ переходит в $\log ^{*} s$. Таким образом можно получить формально несколько более сильное утверждение, чем теорема 3. Но вся необходимая информация содержится и в самой теореме 3. Надо только правильно выбирать нормировку.

Подчеркнем, что условие $\mathscr{L}\left(\zeta_{k}\right) \in \mathscr{A}_{d}(\tau), k=1, \ldots, s$, налагается в теореме 3 на суммы по блокам слагаемых. Для выполнения этого условия достаточно 
(но не необходимо!) потребовать, чтобы $\mathscr{L}\left(X_{j}\right) \in \mathscr{A}_{d}(\tau), j=1, \ldots, n$. Легко строятся примеры, в которых распределения некоторых слагаемых не принадлежат, а распределения сумм - принадлежат классу $\mathscr{A}_{d}(\tau)$.

Схема доказательства теорем 2 и 3 очень близка к соответствующим рассуждениям А. И. Саханенко [72] и У. Айнмаля [31]. При доказательстве теоремы 2 мы предполагаем, что гауссовские векторы $Y_{1}, \ldots, Y_{n}, n=2^{N}$, уже построены, и строим независимые векторы, которые ограничены с вероятностью единица, имеют достаточно гладкие распределения и те же моменты первого, второго и третьего порядка, что исходные независимые случайные векторы $X_{1}, \ldots, X_{n}$. При построении мы используем диадическую схему, предложенную KMT [50]. На первом шаге мы строим сумму $2^{N}$ слагаемых с помощью квантильного преобразования Розенблатта [68] для условных распределений. Затем мы строим суммы по блокам из $2^{N-1}, 2^{N-2}, \ldots, 1$ слагаемых. Точность приближения удается удачно оценивать за счет того, что для гладких распределений слагаемых соответствующие условные распределения также являются гладкими и близкими к гауссовским. После этого мы строим векторы $X_{1}, \ldots, X_{n}$ в несколько этапов. После каждого шага количество непостроенных $X_{k}$ становится меньше в $2^{p}$ раз, где $p$ - подходящим образом выбранное натуральное число. На каждом шаге мы начинаем с уже построенных векторов, которые ограничены с вероятностью единица, имеют достаточно гладкие распределения и требуемые моменты до третьего порядка. Затем мы строим такие независимые векторы, что в каждом блоке из $2^{p}$ слагаемых только первый вектор имеет гладкое распределение с ограниченным носителем. Остальные $2^{p}-1$ векторов имеют требуемые распределения $\mathscr{L}\left(X_{k}\right)$. Эти $2^{p}-1$ векторов из каждого блока берутся в качестве $X_{k}$ и не участвуют в следующих шагах процедуры. При оценке близости случайных векторов на этапах процедуры, описанной выше, мы существенно используем свойства классов $\mathscr{A}_{d}(\tau)$. Совпадение третьих моментов позволяет использовать более точные оценки близости квантилей условных распределений, содержащиеся в работе автора [93]. Идея выбирать в одномерном случае вспомогательные распределения с совпадающими тремя, а не двумя моментами принадлежит Саханенко [72]. Айнмаль [31] ограничился при построении распределениями, у которых совпадают с исходными только моменты первого и второго порядка, не вводя в рассмотрение вспомогательных векторов с требуемыми третьими моментами.

Доказательство теоремы 3 сложнее доказательства теоремы 2 из-за того, что ковариационные операторы слагаемых могут быть различными. Используемые оценки погрешности при применении квантильного преобразования Розенблатта, полученные в [93], доказаны при предположении о том, что каждая вновь построенная случайная величина не коррелирует со всеми уже построенными величинами. Это существенно усложняет и схему доказательства, и вид получаемых оценок.

\section{3. Свойства классов $\mathscr{A}_{d}(\tau)$}

Рассмотрим элементарные свойства классов $\mathscr{A}_{d}(\tau)$, которые существенно используются в доказательстве теорем 2 и 3 (см. [89], [91], [93], [95], [96]). Легко видеть, что если $\tau_{1}<\tau_{2}$, то $\mathscr{A}_{d}\left(\tau_{1}\right) \subset \mathscr{A}_{d}\left(\tau_{2}\right)$. Кроме того, класс $\mathscr{A}_{d}(\tau)$ замкнут 
относительно операции свертки: если $F_{1}, F_{2} \in \mathscr{A}_{d}(\tau)$, то $F_{1} F_{2}=F_{1} * F_{2} \in \mathscr{A}_{d}(\tau)$. Произведения и степени мер понимаются в смысле свертки.

Пусть $\tau \geqslant 0, F=\mathscr{L}(\xi) \in \mathscr{A}_{d}(\tau), y \in \mathbb{R}^{m}$ и $\mathbb{A}: \mathbb{R}^{d} \rightarrow \mathbb{R}^{m}$ - некоторый линейный оператор. Тогда

$$
\mathscr{L}(\mathbb{A} \xi+y) \in \mathscr{A}_{m}(\|\mathbb{A}\| \tau), \quad \text { где }\|\mathbb{A}\|=\sup _{x \in \mathbb{R}^{d},\|x\| \leqslant 1}\|\mathbb{A} x\| .
$$

Предположим, что $\tau \geqslant 0$ и $F_{k}=\mathscr{L}\left(\xi^{(k)}\right) \in \mathscr{A}_{d_{k}}(\tau)$, причем случайные векторы $\xi^{(k)}, k=1,2$, независимы. Пусть $\xi \in \mathbb{R}^{d_{1}+d_{2}}-$ вектор с такими же первыми $d_{1}$ координатами, как у вектора $\xi^{(1)}$, и с такими же последними $d_{2}$ координатами, как у вектора $\xi^{(2)}$. Тогда $F=\mathscr{L}(\xi) \in \mathscr{A}_{d_{1}+d_{2}}(\tau)$. Ясно, что это свойство легко обобщается на любое конечное число независимых векторов $\xi^{(j)}$.

Классы $\mathscr{A}_{d}(\tau)$ тесно связаны с другими естественно определяемыми классами многомерных распределений. Из определения $\mathscr{A}_{d}(\tau)$ следует, что если $\mathscr{L}(\xi) \in \mathscr{A}_{d}(\tau)$, то $\xi$ имеет конечные экспоненциальные моменты $\mathrm{E} e^{\langle h, \xi\rangle}<\infty$ при $h \in \mathbb{R}^{d},\|h\| \tau<1$. Это приводит к экспоненциальному убыванию хвостов распределений.

Условие $\mathscr{L}(\xi) \in \mathscr{A}_{1}(\tau)$ эквивалентно условию В. А. Статулявичуса [83] на скорость роста кумулянтов $\gamma_{m}$ случайной величины $\xi$ :

$$
\left|\gamma_{m}\right| \leqslant \frac{1}{2} m ! \tau^{m-2} \gamma_{2}, \quad m=3,4, \ldots
$$

Эта эквивалентность означает, что если одно из этих условий выполняется с параметром $\tau$, то второе справедливо с параметром $c \tau$, где $c$ - некоторая положительная абсолютная константа. Заметим, впрочем, что условие $\mathscr{L}(\xi) \in \mathscr{A}_{d}(\tau)$ существенно отличается от других многомерных аналогов условия Статулявичуса, рассмотренных Р. Рудзкисом [71] и Л. Саулисом [79].

Другой класс распределений, обозначаемый $\widetilde{\mathscr{A}_{d}}(\tau), \tau \geqslant 0$, упоминался в работе [96]. Он определяется аналогично $\mathscr{A}_{d}(\tau)$ с заменой $(7)$ на

$$
\left|d_{v}^{2} \varphi(z)\right| \leqslant 2\langle\mathbb{D} v, v\rangle
$$

для всех $v \in \mathbb{R}^{d}$ и $\|z\| \tau<1$. То, что классы $\widetilde{\mathscr{A}_{d}}(\tau)$ и $\mathscr{A}_{d}(\tau)$ также эквивалентны, легко проверяется с использованием неравенств Коши. Определение классов $\widetilde{\mathscr{A}_{d}}(\tau)$ в некотором смысле выглядит даже более естественно, чем определение классов $\mathscr{A}_{d}(\tau)$. Константу 2 в определении (14) можно заменить на любую другую константу $C, 1<C<\infty$, отделенную от 1 и от бесконечности. В результате также получатся эквивалентные классы.

Очевидно, что класс $\mathscr{A}_{d}(0)$ совпадает с классом всех $d$-мерных гауссовских распределений. Следующее неравенство было доказано в работе автора [89] и может рассматриваться как оценка устойчивости этой характеризации:

$$
\text { если } F \in \mathscr{A}_{d}(\tau) \text {, то } \pi(F, \Phi(F)) \leqslant c d^{2} \tau \log ^{*}\left(\tau^{-1}\right) \text {, }
$$

где $\pi(\cdot, \cdot)$ - расстояние Прохорова, определенное в работе [62], а $\Phi(F)$ обозначает гауссовское распределение, среднее и ковариационный оператор которого 
такие же, как у $F$. Расстояние Прохорова между распределениями $F, G$ может быть определено формулой

$$
\pi(F, G)=\inf \{\lambda: \pi(F, G, \lambda) \leqslant \lambda\},
$$

где

$$
\pi(F, G, \lambda)=\sup _{Y} \max \left\{F\{Y\}-G\left\{Y^{\lambda}\right\}, G\{Y\}-F\left\{Y^{\lambda}\right\}\right\}, \quad \lambda>0,
$$

а $Y^{\lambda}=\left\{y \in \mathbb{R}^{d}: \inf _{x \in Y}\|x-y\|<\lambda\right\}$ есть $\lambda$-окрестность борелевского множества $Y$ (см. [10], [12]).

В работе автора [89] было также установлено, что

$$
\text { если } F \in \mathscr{A}_{d}(\tau), \text { то } \pi(F, \Phi(F), \lambda) \leqslant c d^{2} \exp \left(-\frac{\lambda}{c d^{2} \tau}\right), \lambda>0 .
$$

Здесь существенно то, что неравенство (16) доказано для всех $\tau>0$ и для произвольного $\operatorname{cov} F$, в отличие от теорем 2 и 3 , в которых $\tau \geqslant 1$ и ковариационные операторы удовлетворяют условию (13). Вопрос о необходимости условия (13) в теоремах 2 и 3 остается открытым.

В работах автора [87], [88] и [90] неравенства (15) и (16) (с заменой $d^{2}$ на $\left.d^{5 / 2}\right)$ были доказаны для сверток распределений из класса $\mathscr{B}_{d}(\tau)$, где $\tau>0$ и

$$
\begin{gathered}
\mathscr{B}_{d}(\tau)=\left\{F=\mathscr{L}(\xi): \mathrm{E} \xi=0,\left|\mathrm{E}\langle\xi, v\rangle^{2}\langle\xi, u\rangle^{m-2}\right| \leqslant \frac{1}{2} m ! \tau^{m-2}\|u\|^{m-2} \mathrm{E}\langle\xi, v\rangle^{2}\right. \\
\text { при всех } \left.u, v \in \mathbb{R}^{d}, m=3,4, \ldots\right\},
\end{gathered}
$$

удовлетворяющих многомерным аналогам условий неравенства Бернштейна. Условие Саханенко $\mathscr{L}(\xi) \in \mathscr{S}_{1}(\tau)$ эквивалентно условию $\mathscr{L}(\xi) \in \mathscr{B}_{1}(\tau)$. Заметим, что если $F\left\{\left\{x \in \mathbb{R}^{d}:\|x\| \leqslant \tau\right\}\right\}=1$, то $F \in \mathscr{B}_{d}(\tau)$.

Сформулируем соотношения между классами $\mathscr{A}_{d}(\tau)$ и $\mathscr{B}_{d}(\tau)$. Обозначим через $\sigma^{2}(F)$ максимальное собственное число ковариационного оператора распределения $F$. Тогда:

а) если $F=\mathscr{L}(\xi) \in \mathscr{B}_{d}(\tau)$, то $\sigma^{2}(F) \leqslant 12 \tau^{2}, \mathrm{E} \xi=0$ и $F \in \mathscr{A}_{d}(c \tau)$;

б) если $F=\mathscr{L}(\xi) \in \mathscr{A}_{d}(\tau), \sigma^{2}(F) \leqslant \tau^{2}$ и $\mathrm{E} \xi=0$, то $F \in \mathscr{B}_{d}(c \tau)$.

Таким образом, грубо говоря, $\mathscr{B}_{d}(\tau)$ образует подкласс распределений $F=$ $\mathscr{L}(\xi-\mathrm{E} \xi), \mathscr{L}(\xi) \in \mathscr{A}_{d}(c \tau)$, для которых $\sigma^{2}(F) \leqslant 12 \tau^{2}$. Неравенства (15) и (16) в этом случае говорят лишь о том, что оба сравниваемых распределения близки к вырожденному закону. Если же $F=\mathscr{L}(\xi) \in \mathscr{A}_{d}(\tau)$ и $\sigma^{2}(F)$ существенно больше, чем $\tau^{2}$, то $\mathscr{L}(\xi / \sigma(F)) \in \mathscr{A}_{d}(\tau / \sigma(F))$ и неравенства (15) и (16) отражают близость распределения $F$ к соответствующему гауссовскому закону.

Если $F$ - безгранично делимое распределение со спектральной мерой, сосредоточенной на шаре $\left\{x \in \mathbb{R}^{d}:\|x\| \leqslant \tau\right\}$, то $F \in \mathscr{A}_{d}(c \tau)$, где $c$ - некоторая положительная абсолютная константа. В работе [89] можно найти и менее ограничительные условия на спектральную меру, обеспечивающие принадлежность безгранично делимого распределения классу $\mathscr{A}_{d}(c \tau)$. 
Пусть $F=\mathscr{L}(\xi) \in \mathscr{A}_{d}(\tau)$ и $\|h\| \tau<1, h \in \mathbb{R}^{d}$. Определим распределения $F_{h}$ равенством $F_{h}\{d x\}=e^{\langle h, x\rangle} F\{d x\} / \mathrm{E} e^{\langle h, \xi\rangle}$. Распределения $F_{h}$ иногда называют преобразованиями Крамера. В доказательстве теорем 2 и 3 распределения $F_{h}$ используются при оценивании вероятностей больших уклонений, соответствующих условным плотностям. Еще одним важным свойством классов $\mathscr{A}_{d}(\tau)$ является то, что $F_{h} \in \mathscr{A}_{d}(2 \tau)$ при $\|h\| \tau \leqslant 1 / 2$. Это дает возможность систематически применять результаты, полученные для исходных распределений, к их преобразованиям Крамера и за счет этого уточнять оценки.

По теореме Штрассена-Дадли (см. работу Р. Дадли [27]) и согласно неравенству (16), для любого распределения $F \in \mathscr{A}_{d}(\tau)$ можно построить на одном вероятностном пространстве случайные векторы $\xi$ и $\eta$ с $\mathscr{L}(\xi)=F$ и $\mathscr{L}(\eta)=\Phi(F)$ так, что

$$
\mathrm{P}\{\|\xi-\eta\|>\lambda\}=\pi(F, \Phi(F), \lambda) \leqslant c d^{2} \exp \left(-\frac{\lambda}{c d^{2} \tau}\right) .
$$

Для сверток ограниченных мер этот факт был использован (иногда с заменой $d^{2}$ на $\left.d^{5 / 2}\right)$, например, в работах [5], [15], [33], [35], [39], [67].

Подчеркнем, что теорема Штрассена-Дадли гарантирует существование построения с равенством в (18) только при фиксированном $\lambda$. Пример, показывающий невозможность построения с равенством в (18) при всех $\lambda$ одновременно, можно найти в обзоре [10]. Теорема Штрассена-Дадли дает возможность из оценок для $\pi(F, G, \lambda)$ автоматически получать утверждения типа неравенства (18). Первоначальное доказательство Штрассена [85] было неконструктивным. Дадли [27] дал сложное конструктивное доказательство, основанное на комбинаторных идеях. Наконец, Г. Шай [80] нашел короткое доказательство, основанное на теореме о двойственности.

Упомянем, наконец, результат о сильной аппроксимации сумм независимых случайных векторов безгранично делимыми распределениями. Следующая теорема 4 вытекает из основного результата работы автора [92] и теоремы Штрассена-Дадли. Неравенство (19) можно рассматривать как обобщение оценки (18) на свертки распределений с неограниченными носителями.

Теорема 4. Предположим, что $d$-мерные распределения $F_{i}, i=1, \ldots, n$, представлены в виде смесей вероятностных распределений $U_{i} u V_{i}$ :

$$
F_{i}=\left(1-p_{i}\right) U_{i}+p_{i} V_{i}
$$

¿əe

$$
0 \leqslant p_{i} \leqslant 1, \quad \int x U_{i}\{d x\}=0, \quad U_{i}\left\{\left\{x \in \mathbb{R}^{d}:\|x\| \leqslant \tau\right\}\right\}=1, \quad \tau \geqslant 0,
$$

и $V_{i}$ - произвольные распределения. Тогда для любого фиксированного $\lambda>0$ можно построить на одном вероятностном пространстве случайные векторы $\xi$ и $\eta$ mak, чmo

$$
\mathrm{P}\{\|\xi-\eta\|>\lambda\} \leqslant c(d)\left(\max _{1 \leqslant i \leqslant n} p_{i}+\exp \left(-\frac{\lambda}{c(d) \tau}\right)\right)+\sum_{i=1}^{n} p_{i}^{2}
$$

$u$

$$
\mathscr{L}(\xi)=\prod_{i=1}^{n} F_{i}, \quad \mathscr{L}(\eta)=\prod_{i=1}^{n} e\left(F_{i}\right)
$$


где $c(d)$ зависит только от $d$, a $e\left(F_{i}\right)$ обозначает безгранично делимое обобщенное распределение Пуассона с характеристической функиией $\exp \left(\widehat{F}_{i}(t)-1\right)$, где $\widehat{F}_{i}(t)=\int e^{i t x} F_{i}\{d x\}$. Если распределения $V_{i}$ одинаковь, слагаемое $\sum_{i=1}^{n} p_{i}^{2}$ в (19) можно опустить.

Утверждение теоремы 4 остается в силе также при

$$
\mathscr{L}(\eta)=\Phi\left(\prod_{i=1}^{n}\left(\left(1-p_{i}\right) U_{i}+p_{i} E\right)\right) \prod_{i=1}^{n} e\left(\left(1-p_{i}\right) E+p_{i} V_{i}\right)
$$

где $E$ - распределение, сосредоточенное в нуле.

\section{4. Точность аппроксимации в принципе инвариантности Штрассена}

В этом разделе мы дадим обзор результатов, полученных при решении задачи (А) для независимых одинаково распределенных одномерных и многомерных случайных векторов $X_{j}$ с $\mathscr{L}\left(X_{j}\right)=\mathscr{L}(X)$ и с конечными моментами $\mathrm{E} H(\|X\|)<\infty$, где $H(x)$ - монотонная функция, растущая не медленнее, чем $x^{2+\delta}$, и не быстрее, чем $e^{c x}$ (см. теорему 5 ниже). Приближающие векторы $Y_{j}$ имеют те же средние и ковариационные операторы, что и приближаемые векторы $X_{j}$.

$\Phi$. Штрассен [84] начал изучение задачи (А) в одномерном случае. Он показал, что существует такое построение, что

$$
\left\|\sum_{j=1}^{n} X_{j}-\sum_{j=1}^{n} Y_{j}\right\|=o(\sqrt{n \log \log n})
$$

предполагая лишь выполнение условий $\mathrm{E} X=0$ и $\mathrm{E} X^{2}<\infty$. Многомерный вариант этого утверждения содержится в работе У. Филиппа [61].

Усиления и обобщения соотношения (20) при дополнительных предположениях о существовании моментов были получены в работах [16], [20], [46] и [86].

Штрассен [84], [86] использовал при построении вложение Скорохода (см. [82]). Длительное время казалось, что метод Скорохода дает оптимальные по порядку оценки. Штрассен [86] получил оценку порядка $O\left((n \log \log n)^{1 / 4}(\log n)^{1 / 2}\right)$ при $\mathrm{E} X^{4}<\infty$ и выдвинул гипотезу о том, что дополнительные моментные предположения не могут существенно улучшить точность аппроксимации и если эта точность записывается как $o\left((n \log \log n)^{1 / 4}(\log n)^{1 / 2}\right)$, то распределения слагаемых гауссовские. Дж. Кифер [48] показал, что это действительно так, если пользоваться методом вложения Скорохода. Используя квантильные преобразования, М. Чёрге и П. Ревес [20] опровергли упомянутую выше гипотезу, показав, что для любого $0<\varepsilon<1 / 2$ можно выписать моментные условия, обеспечивающие точность аппроксимации $o\left(n^{\varepsilon}\right)$.

В одномерном случае правильные по порядку результаты были получены в работах KMT [50] и П. Майора [53]. В частности, было показано, что если 
$\mathrm{E} X=0$ и $\mathrm{E}|X|^{\gamma}<\infty$ для некоторого $\gamma>2$, то существует такое построение, что

$$
\left\|\sum_{j=1}^{n} X_{j}-\sum_{j=1}^{n} Y_{j}\right\|=o\left(n^{1 / \gamma}\right) .
$$

KMT [50] рассмотрели случай $\gamma>3$, а П. Майор [53] получил результат для $2<\gamma \leqslant 3$. Соответствующее многомерное утверждение было доказано У. Айнмалем [31]. Случай $2<\gamma \leqslant 3$ был исследован ранее Э. Бергером [3], см. также работу А. И. Саханенко [76].

Для неотрицательных чисел $\delta$ и $x_{0}$ введем класс $\mathscr{H}\left(\delta, x_{0}\right)$ таких неотрицательных неубывающих функций $H:[0, \infty) \rightarrow \mathbb{R}^{1}$, что функции $H(x) / x^{2+\delta}$ и $x / \log H(x)$ не убывают при $x \geqslant x_{0}$.

Пусть $H \in \mathscr{H}\left(\delta, x_{0}\right)$. Тогда функции $H^{-1}(y) / y^{1 /(2+\delta)}$ и $(\log y) / H^{-1}(y)$ не возрастают при $y \geqslant H\left(x_{0}\right)$. Более того,

$$
\frac{x^{2+\delta} H\left(x_{0}\right)}{x_{0}^{2+\delta}} \leqslant H(x) \leqslant \exp \left(\frac{x \log H\left(x_{0}\right)}{x_{0}}\right) \quad \text { при } x \geqslant x_{0}
$$

и

$$
\frac{x_{0} \log y}{\log H\left(x_{0}\right)} \leqslant H^{-1}(y) \leqslant \frac{y^{1 /(2+\delta)} x_{0}}{\left(H\left(x_{0}\right)\right)^{1 /(2+\delta)}} \quad \text { при } y \geqslant H\left(x_{0}\right) .
$$

Здесь и в дальнейшем $H^{-1}(\cdot)$ будет обозначать обратную функцию для функции $H$. Обозначим $\mathscr{H}=\bigcup_{\delta>0, x_{0}>0} \mathscr{H}\left(\delta, x_{0}\right)$.

TeOpema 5. Пусть $H \in \mathscr{H}$ u $X$ - случайный вектор с $\mathrm{E} X=0, \operatorname{cov} X=\mathbb{I}_{d}$ u $\mathrm{E} H(\|X\|)<\infty$. Тогда существует такое построение, что

$$
\mathrm{P}\left\{\limsup _{n \rightarrow \infty} \frac{1}{H^{-1}(n)}\left\|\sum_{j=1}^{n} X_{j}-\sum_{j=1}^{n} Y_{j}\right\| \leqslant C\right\}=1,
$$

где $C<\infty$ - неслучайная величина, зависящая только от $d, \mathscr{L}(X)$ и от функиии $H(\cdot)$.

Легко видеть, что из (24) следует, что

$$
\left\|\sum_{j=1}^{n} X_{j}-\sum_{j=1}^{n} Y_{j}\right\|=O\left(H^{-1}(n)\right) \quad \text { п.н. при } n \rightarrow \infty \text {. }
$$

Теорема 5, доказанная в работе автора [101], обобщает на многомерный случай результаты KMT [50] и Майора [53]. Ясно, что утверждение теоремы 5 остается справедливым без предположения $\operatorname{cov} X=\mathbb{I}_{d}$.

В одномерном случае то же самое утверждение было доказано в работе KMT [50] для функций $H$ из класса $\widetilde{\mathscr{H}}\left(\delta, x_{0}\right), \delta>0$, таких неотрицательных неубывающих функций $H \in \mathscr{H}\left(\delta, x_{0}\right)$, что функции $H(x) / x^{3+\delta}$ не убывают при $x \geqslant x_{0}$. Майор [53] распространил результат на такие функции $H$ из класса $\mathscr{H}\left(\delta, x_{0}\right), \delta>0$, что функции $H(x) / x^{3}$ не возрастают. Бергер [3] обобщил результат Майора [53] на многомерный случай. 
Айнмаль [31] доказал то же самое утверждение для функций $H$ из класса $\mathscr{H}^{*}\left(\delta, x_{0}\right), \delta>0$, таких неотрицательных неубывающих функций $H$, что функции $H(x) / x^{3+\delta}$ и $\sqrt{x} / \log H(x)$ не убывают при $x \geqslant x_{0}$. Ясно, что существуют функции, которые принадлежат $\mathscr{H}\left(\delta, x_{0}\right)$ и не принадлежат $\mathscr{H}^{*}\left(\delta, x_{0}\right)$. В качестве примера можно привести функции $H(x)=x^{\gamma}, 2<\gamma \leqslant 3$, и $H(x)=\exp \left(\lambda x^{\beta}\right), 1 / 2<\beta \leqslant 1, \lambda>0$. Тем самым, в теореме 5 обобщаются результаты Айнмаля [31]. В частности, в теореме 5 содержится результат для функций $H \in \mathscr{H}$, которые могут расти быстрее, чем $e^{c \sqrt{x}}$.

Утверждение теоремы 5 оптимально в следующем смысле: если

$$
\mathrm{E} H(\|X\|)=\infty
$$

в условиях теоремы 5 , то

$$
\mathrm{P}\left\{\limsup _{n \rightarrow \infty} \frac{1}{H^{-1}(n)}\left\|\sum_{j=1}^{n} X_{j}-\sum_{j=1}^{n} Y_{j}\right\| \geqslant \frac{1}{4}\right\}=1
$$

для любого построения независимых одинаково распределенных случайных векторов $X_{j}$ и независимых гауссовских случайных векторов $Y_{j}$ с требуемыми распределениями на одном вероятностном пространстве. Действительно, условие (26) эквивалентно тому, что

$$
\sum_{j=1}^{\infty} \mathbf{P}\left\{\|X\|>H^{-1}(j)\right\}=\infty .
$$

По лемме Бореля-Кантелли, с вероятностью единица бесконечно много $X_{j}$ удовлетворяют неравенству $\left\|X_{j}\right\|>H^{-1}(j)$. С другой стороны, согласно (23),

$$
\sum_{j=1}^{\infty} \mathrm{P}\left\{\left\|Y_{j}\right\|>\frac{H^{-1}(j)}{2}\right\}<\infty .
$$

Согласно лемме Бореля-Кантелли, с вероятностью единица лишь конечное число $Y_{j}$ удовлетворяет неравенству $\left\|Y_{j}\right\|>H^{-1}(j) / 2$. Поэтому для бесконечного множества чисел $n$

$$
\frac{1}{H^{-1}(n)}\left\|\sum_{j=1}^{n} X_{j}-\sum_{j=1}^{n} Y_{j}\right\| \geqslant \frac{1}{4} .
$$

Отсюда вытекает (27). Это рассуждение принадлежит Л. Брейману [16], см. также теорему 5.3 из работы Майора [55].

Утверждение теоремы 1 может не выполняться для $H \in \mathscr{H}\left(0, x_{0}\right)$. Брейман [16] показал, что утверждение (21), вообще говоря, неверно для $\gamma=0$. Майор [54] установил, что для любой последовательности $\left\{a_{n}\right\}$ вещественных чисел, для которой $a_{n} \nearrow \infty$, существует такое одномерное распределение $\mathscr{L}(X)$ с $\mathrm{E} X^{2}<\infty, \mathrm{E} X=0$, что для любого построения, удовлетворяющего (1),

$$
\mathrm{P}\left\{\limsup _{n \rightarrow \infty} a_{n}(n \log \log n)^{-1 / 2}\left\|\sum_{j=1}^{n} X_{j}-\sum_{j=1}^{n} Y_{j}\right\|=\infty\right\}=1 .
$$

Это подтверждает оптимальность результата Штрассена (20). 
Случай, когда $H(x) / x^{2} \rightarrow \infty$ и $H(x) / x^{2+\delta} \rightarrow 0$ для любого $\delta>0$, был исследован Айнмалем [30] как в одномерном, так и в многомерном случае. Для $H \in \mathscr{H}\left(0, x_{0}\right)$ точность аппроксимации может быть существенно улучшена, если мы заменим $Y_{j}$ с ковариационными операторами, совпадающими с ковариационными операторами $X_{j}$, на $Y_{j}$ с ковариационными операторами урезанных векторов. См. работы Майора [54], [56], Айнмаля [30], [32], а также Айнмаля и Мейсона [34]. История вопроса изложена в статье Айнмаля [32].

Доказательство теоремы 5 основано на теореме 2, доказанной автором в [95]. Следствие 2 является частным случаем теоремы 5 при $H(x)=e^{\lambda x}, \lambda>0$.

Заметим, что если условия теоремы 5 выполнены при $H(x)=|x|^{\gamma}$, то удается обеспечить выполнение соотношения (21), которое сильнее, чем (25). С другой стороны, Шао [63] показал, что для функций $H(x)=e^{x^{\beta}}, 0<\beta<1$, невозможно заменить $O$ большое на $о$ малое в соотношении (25), по крайней мере для тех распределений $\mathscr{L}(X)$, для которых $\mathrm{E} e^{2\|X\|^{\beta}}=\infty$. В работе [63] отмечается, что это легко следует из приведенного выше рассуждения Бреймана [16] (см. (26) и (27)). В этом случае (25) превращается в

$$
\left\|\sum_{j=1}^{n} X_{j}-\sum_{j=1}^{n} Y_{j}\right\|=O\left((\log n)^{1 / \beta}\right) \quad \text { п.н. при } n \rightarrow \infty .
$$

Остается открытым вопрос об описании класса функций $H \in \mathscr{H}$, для которых можно заменить $O$ большое на $о$ малое в соотношении $(25)$. При $H(x)=x^{\gamma}$, $\gamma>2$, это так, т. е. $O\left(H^{-1}(n)\right)=O\left(n^{1 / \gamma}\right)$ можно заменить на $o\left(n^{1 / \gamma}\right)$.

Доказательство теоремы 5 представляет собой слегка модифицированный вариант соответствующего доказательства из работы Айнмаля [31]. Он построил доказательство на основе собственного результата для векторов с конечными экспоненциальными моментами. Использование более общей теоремы 2 дает возможность получить более общий результат теоремы 5. Еще одно отличие состоит в том, что в теореме 2 фигурируют распределения из класса $\mathscr{A}_{d}(\tau)$, определение которого отличается от класса распределений $\mathscr{S}_{d}(\tau)$, рассмотренного Айнмалем в работе [31]. Учитывается также то, что распределения урезанных векторов принадлежат классам $\mathscr{A}_{d}(\tau)$ с параметром $\tau$, который существенно меньше уровня урезания $u$. Близкая идея использовалась Айнмалем в [31], см. также работы [41] и [100].

\section{5. Точность аппроксимации в задаче (В) для сумм неодинаково распределенных векторов с конечными моментами $\mathrm{E} H\left(\left\|X_{j}\right\|\right)<\infty$}

В этом разделе приведены результаты, опубликованные в работе автора [99]. Они относятся к задаче (В) для сумм неодинаково распределенных векторов с конечными моментами $\mathrm{E} H\left(\left\|X_{j}\right\|\right)<\infty$, где $H(x)$ - монотонная функция, растущая не медленнее, чем $x^{2}$, и не быстрее, чем $e^{c x}$. Их доказательства основаны на теореме 3, в которой рассматриваются векторы с конечными экспоненциальными моментами. Результаты данного раздела дают достаточно хорошее приближение к многомерному обобщению результатов работы А.И. Саханенко [73]. В первую очередь речь идет об обобщении следующего результата. 
Теорема 6 [73]. Пусть $X_{1}, \ldots, X_{n}$ - независимые случайные величины $c$ $\mathrm{E} X_{j}=0, j=1, \ldots, n$. Пyсmь $\gamma>2 u$

$$
L_{\gamma}=\sum_{j=1}^{n} \mathrm{E}\left|X_{j}\right|^{\gamma}<\infty
$$

Тогда существует такое построение, что

$$
\mathrm{E}\left(\Delta_{n}(X, Y)\right)^{\gamma} \leqslant c \gamma^{2 \gamma} L_{\gamma}
$$

u, следовательно, при всех $z>0$

$$
\mathrm{P}\left\{\Delta_{n}(X, Y) \geqslant z\right\} \leqslant c \gamma^{2 \gamma} L_{\gamma} z^{-\gamma}
$$

Кроме того, существуют такие абсолютные положительные постоянные $c_{1}$ $u c_{2}$, чmo

$$
\mathrm{P}\left\{\Delta_{n}(X, Y) \geqslant c_{1} z\right\} \leqslant c_{2} \gamma^{2 \gamma b} b^{\gamma b}\left(L_{\gamma} z^{-\gamma}\right)^{b}+2 \sum_{j=1}^{n} \mathrm{P}\left\{\left|X_{j}\right| \geqslant \frac{z}{b}\right\}
$$

при всех $b \geqslant 1 u z \geqslant 0$.

Следует отметить, что в работе А. И. Саханенко [73] можно найти более общие результаты. Из них следует много других аппроксимационных результатов (см. [64]). После естественной нормировки мы видим, что неравенство (28) эквивалентно неравенству

$$
\mathrm{E}\left(\frac{\Delta_{n}(X, Y)}{\sigma}\right)^{\gamma} \leqslant \frac{c \gamma^{2 \gamma} L_{\gamma}}{\sigma^{\gamma}}
$$

где $\sigma^{2}=\mathrm{D}\left(\sum_{j=1}^{n} X_{j}\right)$. Ясно, что $L_{\gamma} / \sigma^{\gamma}, 2<\gamma \leqslant 3$, представляет собой известную дробь Ляпунова, фигурирующую в неравенствах Ляпунова и Эссеена для равномерного расстояния в центральной предельной теореме. Подчеркнем, что оценка (31) доказана при любых $\gamma>2$, в то время как неравенство Ляпунова может быть неверно при $\gamma>3$.

Аналог неравенства (28) теоремы 6, который справедлив при всех $n$ одновременно на одном и том же вероятностном пространстве, был выведен М. А. Лифшицем [52] из теоремы 6 с помощью одномерного неравенства Розенталя [69], [70], см. также [47].

Следуя работе А.И. Саханенко [73], введем класс $\mathscr{G}(u), u \geqslant 0$, таких положительных функций $G(\cdot)$, что функции $G(x)$ и $x / \log G(x)$ не убывают при $x>u$. Например, функции $G(x)=x^{\gamma}, \gamma>0$, и $\exp \left(\lambda x^{\beta}\right), 0 \leqslant \beta \leqslant 1, \lambda>0$, принадлежат $\mathscr{G}(e)$ и $\mathscr{G}(0)$ соответственно.

Для произвольного случайного вектора $\xi$ в разделах 5 и 6 мы будем обозначать $\xi^{[u]}$ его срезку на уровне $u>0$ :

$$
\xi^{[u]}= \begin{cases}\xi, & \text { если }\|\xi\| \leqslant u, \\ 0 & \text { в противном случае. }\end{cases}
$$


Положим

$$
\xi^{(u)}=\xi-\xi^{[u]}
$$

Основными результатами данного раздела являются следующая теорема 7 и ее следствия 4-6, опубликованные в работе автора [99].

Теорема 7. Пусть $\alpha, u>0$. Предположим, что $X_{1}, \ldots, X_{n}$ - независимые случайные векторы с $\mathrm{E} X_{j}=0, j=1, \ldots, n, G(\cdot) \in \mathscr{G}(u) u$

$$
F(x)=\sum_{j=1}^{n} \mathrm{P}\left\{\left\|X_{j}\right\|>x\right\} \leqslant \frac{1}{G(x)} \quad \text { для всех } x>u \text {. }
$$

Предположим, что выполнено условие $\mathbf{B}_{s}\left(r^{2} u^{2}, C r^{2} u^{2}\right)$ с некоторыми постоянными $r \geqslant 2$ и $C \geqslant 1$. Пусть

$$
B^{2}(u)=\sum_{j=1}^{n} \mathrm{E}\left\|X_{j}^{(u)}\right\|^{2} \leqslant u^{2}
$$

$u$

$$
A(u)=\max _{1 \leqslant k \leqslant n}\left\|\sum_{i=1}^{k} \mathrm{E} X_{i}^{[u]}\right\|=\max _{1 \leqslant k \leqslant n}\left\|\sum_{i=1}^{k} \mathrm{E} X_{i}^{(u)}\right\| .
$$

Тогда существует такое построение, что

$$
\begin{aligned}
& \mathrm{P}\left\{\Delta_{n}(X, Y)>x+x_{1}+x_{2}+A(u)\right\} \leqslant 2 \exp \left(-\frac{x_{2}^{2}}{16 B^{2}(u)}\right) \\
& +\exp \left(-\frac{a_{6} x_{1}}{r u d^{9 / 2} \log ^{*} d}\right)+\mathrm{P}\left\{\max _{1 \leqslant j \leqslant n}\left\|X_{j}\right\|>z\right\}+\frac{e G^{1-x / z}(z)}{G(u)}
\end{aligned}
$$

для всех $x \geqslant z \geqslant u>0$, если $x_{1}>a_{7} r u d^{15 / 2+\alpha} \log ^{*} d \cdot \log ^{*} s u x_{2}>c B(u) \sqrt{d}$, где $a_{6}, a_{7}$ - некоторые положительные величинь, зависящие только от $\alpha$ u $C$, а с-некоторая абсолютная постоянная.

СЛЕДСТвИЕ 4. Пусть выполнены условия теоремы 7 с $G(x)=H(x) / L_{H}$, где $H:[0, \infty) \rightarrow \mathbb{R}^{1}$ - такая неотрицательная неубывающая функиия, что $H(0)=0$,

$$
L_{H}=\sum_{j=1}^{n} \mathrm{E} H\left(\left\|X_{j}\right\|\right) \leqslant \frac{H(u)}{e} .
$$

Тогда утверждение теоремы 7 справедливо с

$$
\mathrm{P}\left\{\Delta_{n}(X, Y)>5 z\right\} \leqslant \frac{2 L_{H}}{H(z)}+\exp \left(-\frac{a_{8} z}{r u d^{9 / 2} \log ^{*} d}\right),
$$

если $z>a_{9} r u d^{15 / 2+\alpha} \log ^{*} d \cdot \log ^{*} s>0$, где $a_{8}, a_{9}$ - некоторые положителъные величины, зависящие только от $\alpha и$ с. 
СлеДСТвие 5. Пусть $\alpha>0$ и $H:[0, \infty) \rightarrow \mathbb{R}^{1}-$ такая неотрицательная функция, что $H(0)=0$, функция $H(x) / x^{2}$ не убывает и функция $H(x) / x^{\lambda}$ не возрастает при некотором $\lambda \geqslant 2$. Предположим, что $X_{1}, \ldots, X_{n}$ - независимые случайные векторы с $\mathrm{E} X_{j}=0, j=1, \ldots, n, u L_{H}<\infty$, где величина $L_{H}$ определена в (38). Положим

$$
K_{H}=H^{-1}\left(e^{\lambda} L_{H}\right)
$$

Предположим, что выполнено условие $\mathbf{B}_{s}\left(r^{2} K_{H}^{2}, C r^{2} K_{H}^{2}\right)$ с некоторыми постоянными $r \geqslant 2$ и $C \geqslant 1$. Тогда существует такое построение, что при $z>a_{9} d^{15 / 2+\alpha} \log ^{*} d \cdot r K_{H} \log ^{*} s$

$$
\mathrm{P}\left\{\Delta_{n}(X, Y)>5 z\right\} \leqslant \frac{2 L_{H}}{H(z)}+\exp \left(-\frac{a_{8} z}{r K_{H} d^{9 / 2} \log ^{*} d}\right)
$$

где $a_{8}, a_{9}$ - положительные величины, зависящие только от $\alpha$ и $C$ (те же, что в следствии 4).

СлеДСТвиЕ 6. Предположим, что $\alpha>0$ u $X_{1}, \ldots, X_{n}$-независимые $d$-мерные случайные векторы с $\mathrm{E} X_{j}=0, j=1, \ldots, n$. Пусть $\gamma \geqslant 2 u$

$$
L_{\gamma}=\sum_{j=1}^{n} \mathrm{E}\left\|X_{j}\right\|^{\gamma}<\infty
$$

Предположим, что выполнено условие $\mathbf{B}_{s}\left(r^{2} L_{\gamma}^{2 / \gamma}, C r^{2} L_{\gamma}^{2 / \gamma}\right)$ с некоторыми постоянными $r \geqslant 2$ е и $C \geqslant 1$. Тогда существует такое построение, что при $z>a_{9} d^{15 / 2+\alpha} \log ^{*} d \cdot r L_{\gamma}^{1 / \gamma} \log ^{*} s$

$$
\mathrm{P}\left\{\Delta_{n}(X, Y) \geqslant 5 z\right\} \leqslant 2 L_{\gamma} z^{-\gamma}+\exp \left(-\frac{a_{8} z}{r L_{\gamma}^{1 / \gamma} d^{9 / 2} \log ^{*} d}\right)
$$

где $a_{8}, a_{9}$ - положительные величины, зависящие только от $\alpha$ и $C$ (те же, что в следствии 4).

Теорема 7 и следствия 4 и 6 являются многомерными аналогами несколько ослабленных вариантов одномерных результатов А. И. Саханенко [73] (см. неравенство (29) теоремы 6). В частности, теорему 7 можно рассматривать как аналог теоремы 4 из статьи А. И. Саханенко [73].

Грубо говоря, в теореме 6 та же формулировка, что в следствии 6 , но без экспоненциального слагаемого в правой части (41), без условия $\mathbf{B}_{s}\left(r^{2} L_{\gamma}^{2 / \gamma}, C r^{2} L_{\gamma}^{2 / \gamma}\right)$ и без каких бы то ни было ограничений на $z>0$. Если $\mathrm{D}\left(\sum_{j=1}^{n} X_{j}\right) \geqslant 32 L_{\gamma}^{2 / \gamma}$, то условие $\mathbf{B}_{s}\left(r^{2} L_{\gamma}^{2 / \gamma}, C r^{2} L_{\gamma}^{2 / \gamma}\right)$ всегда выполняется в одномерной ситуации, поскольку $\mathrm{D} X_{j} \leqslant\left(\mathrm{E}\left|X_{j}\right|^{\gamma}\right)^{2 / \gamma} \leqslant L_{\gamma}^{2 / \gamma}, j=1, \ldots, n$, и мы всегда можем найти такие $m_{1}, \ldots, m_{s}$, что $\mathbf{B}_{s}\left(r^{2} L_{\gamma}^{2 / \gamma}, C r^{2} L_{\gamma}^{2 / \gamma}\right)$ справедливо с $e^{-1} r=C=2$. Заметим, что при $z \leqslant L_{\gamma}^{1 / \gamma}$ неравенство (41) заведомо выполнено. Очевидно, что в случае $d=1, e^{-1} r=C=2$ экспоненциальный член может быть легко оценен сверху 
величиной $c L_{\gamma} z^{-\gamma}$ при $z>c L_{\gamma}^{1 / \gamma}$ с абсолютной положительной постоянной $c$. В вырожденном случае, когда $\mathrm{D}\left(\sum_{j=1}^{n} X_{j}\right) \leqslant 2 L_{\gamma}^{2 / \gamma}$, требуемые оценки справедливы при любом построении случайных векторов $\left\{X_{j}\right\}$ и $\left\{Y_{j}\right\}$ на одном вероятностном пространстве (см. неравенство (63) в разделе 7 ).

Таким образом, отличие от результата А. И. Саханенко состоит только в появлении логарифмического множителя $\log ^{*} s$ в ограничениях на $z$. Ясно, что $\log ^{*} s$ ведет себя как $\log ^{*} n$ в случае независимых одинаково распределенных $X_{1}, \ldots, X_{n}$ и как логарифм дисперсий координат сумм $X_{1}+\cdots+X_{n}$ в общем случае.

Поэтому естественно попытаться установить многомерный аналог неравенства (29), который справедлив без ограничений на $z$, а также многомерные аналоги неравенств (28) и (30). При некоторых дополнительных условиях это сделано в теореме 10, дающей частичное решение проблемы. Кроме того, в теореме 11 получено адекватное многомерное обобщение неравенства (28) в случае одинаково распределенных слагаемых.

Заметим, что если $s r^{2} L_{\gamma}^{2 / \gamma}=1$ в условиях следствия 6 (что соответствует естественной нормировке, при которой последняя сумма имеет дисперсии координат порядка единица), то из неравенства (41) вытекает оценка

$$
\mathrm{P}\left\{\Delta_{n}(X, Y) \geqslant a L_{\gamma}^{1 /(1+\gamma)}\right\} \leqslant a L_{\gamma}^{1 /(1+\gamma)} \quad \text { при } \gamma \geqslant 2,
$$

где $a$ - положительная величина, зависящая только от $d, r, \gamma, \alpha$ и $C$. Ясно, что при естественной нормировке величина $L_{\gamma}^{1 /(\gamma+1)}, 2<\gamma \leqslant 3$, с точностью до ограниченного множителя представляет собой дробь Ляпунова, уже упоминавшуюся при обсуждении теоремы 6. Неравенство (42) дает оценку для расстояния Прохорова порядка $L_{\gamma}^{1 /(\gamma+1)}$ при любых $\gamma \geqslant 2$. Именно такой порядок имеет оптимальная по порядку одномерная оценка, вытекающая из теоремы 6 (см. [10]-[12], [73]).

Следствие 5 является естественным обобщением следствия 6 на случай общих функций "степенного типа" $H(\cdot)$, которые могут не совпадать с $H(x) \equiv$ $|x|^{\gamma}$. Формула (39) для уровня урезания $u=K_{H}$, по-видимому, появилась в работе [99] впервые. А.И. Саханенко [73] применил ее в частном случае $H(x) \equiv|x|^{\gamma}$ (когда $\left.K_{H}=e L_{\gamma}^{1 / \gamma}\right)$.

\section{6. Точность аппроксимации в задаче (В) для сумм одинаково распределенных векторов с конечными моментами $\mathrm{E} H(\|X\|)<\infty$}

В этом разделе приведены результаты, опубликованные в работе автора [100] (см. теоремы 8, 9 и следствие 7). Они относятся к задаче (В) для сумм одинаково распределенных векторов $X_{j}$ с $\mathscr{L}\left(X_{j}\right)=\mathscr{L}(X)$ и с конечными моментами $\mathrm{E} H(\|X\|)<\infty$, где $H(x)$ - монотонная функция, растущая не медленнее, чем $x^{2+\delta}$, и не быстрее, чем $e^{c x}$. Их доказательства основаны на теореме 2. Результаты данного раздела обобщают и уточняют результаты Айнмаля [31]. В работе [31] он получил многомерные результаты в случае, когда 
Е $H(\|X\|)<\infty$, где $H$ - монотонная функция, растущая не медленнее, чем $x^{3+\delta}$, и не быстрее, чем $e^{c \sqrt{x}}$. Следуя Саханенко [73], Айнмаль [31] построил доказательство на основе своего же результата для векторов с конечными экспоненциальными моментами. В этом разделе приведены результаты для функций $H$, которые могут расти быстрее, чем $e^{c \sqrt{x}}$.

Теорема 8 является обобщением теоремы 4 из работы Айнмаля [31], доказавшего то же утверждение для функций $H$ из класса $\mathscr{H}^{*}\left(\delta, x_{0}\right)$ (см. раздел 4). Из теоремы 8 в одномерном случае следуют соответствующие результаты KMT [50].

Tеорема 8. Пусть случайный вектор $X$ maков, что $\mathrm{E} X=0, \operatorname{cov} X=\mathbb{I}_{d}$ u $\mathrm{E} H(\|X\|)<\infty$ для некоторой функиии $H \in \mathscr{H}\left(\delta, x_{0}\right)$ с $x_{0}>0,0<\delta<1 / 2$. Тогда для любых $z>0$ u $n \in \mathbf{N}$ существует такое построение, что

$$
\mathrm{P}\left\{\Delta_{n}(X, Y)>a_{10} z\right\} \leqslant \frac{a_{11} n}{H(z)},
$$

где $a_{10}, a_{11}$ - некоторье положительные величины, зависящие только от $d$, $\mathscr{L}(X)$ и от функиии $H(\cdot)$.

Теорему 9 можно рассматривать как аналог теоремы 7 для случая одинаково распределенных слагаемых. Заметим, впрочем, что между неравенствами (37) и (45) имеются и другие отличия. Теорема 9 является усилением теоремы 8.

Теорема 9. Пусть случайный вектор Х и функиия Н удовлетворяют условиям теоремы 8, а число $и>0$ таково, что

$$
L_{H}=n \mathrm{E} H(\|X\|) \leqslant H(u) .
$$

Пусть $G(\cdot) \in \mathscr{G}(u)$, где

$$
G(x)=\frac{H(x)}{L_{H}} .
$$

Тогда для любого $n \geqslant n_{0}$ существует такое построение, что

$$
\begin{aligned}
& \mathrm{P}\left\{\Delta_{n}(X, Y)>x+x_{1}+x_{2}+A(u)\right\} \leqslant 2 \exp \left(-\frac{n x_{2}^{2}}{32 B^{4}(u)}\right) \\
& \quad+\exp \left(-\frac{a_{12} x_{1} \log H(u)}{u}\right)+\mathrm{P}\left\{\max _{1 \leqslant j \leqslant n}\left\|X_{j}\right\|>z\right\}+\frac{e G^{1-x / z}(z)}{G(u)},
\end{aligned}
$$

если $x \geqslant z \geqslant u>0, x_{1}>a_{13}$ u u $x_{2}>c_{0} B^{2}(u) \sqrt{d} / \sqrt{n}$, где

$$
\begin{aligned}
B^{2}(u) & =n \mathrm{E}\left\|X^{(u)}\right\|^{2} \leqslant u^{2}, \\
A(u) & =n\left\|\mathrm{E} X^{[u]}\right\|=n\left\|\mathrm{E} X^{(u)}\right\|,
\end{aligned}
$$

$a_{12}, a_{13}$ и $n_{0}$ - некоторые положительные величины, зависящие только от $d$, $\mathscr{L}(X)$ и от функции $H(\cdot)$, а $c_{0}$ - некоторая абсолютная постоянная.

СлеДСТВИЕ 7. Пусть выполнены условия теоремы 9 при

$$
u=C_{1} H^{-1}\left(C_{2} L_{H}\right),
$$


где $C_{1} \geqslant 1$ и $C_{2} \geqslant 1$ - некоторые положительные постоянные. Тогда утверждение теоремы 9 справедливо для любого $n \geqslant 1 c$

$$
\mathrm{P}\left\{\Delta_{n}(X, Y)>a_{14} z\right\} \leqslant \frac{a_{15} n}{H(z)}
$$

для любого $z>0$, где $a_{14}$ и $a_{15}$ - некоторые положительные величины, зависящие только от $C_{1}, C_{2}, d, \mathscr{L}(X)$ и от функции $H(\cdot)$.

Легко видеть, что утверждения теоремы 8 и следствия 7 остаются справедливыми без предположения $\operatorname{cov} X=\mathbb{I}_{d}$.

Утверждение следствия 7 гораздо сильнее утверждения теоремы 8, поскольку (49) выполняется при всех $z>0$ одновременно на одном и том же вероятностном пространстве, в то время как в теореме 8 вероятностное пространство зависит от $z$, т. е. (43) может не выполняться для иных значений $z$. С другой стороны, в следствии 7 предполагается выполнение условия $G(\cdot) \in \mathscr{G}(u)$ с $u$ из (48). Существуют функции, которые удовлетворяют условиям теоремы 8 и для которых условие $G(\cdot) \in \mathscr{G}(u)$ не выполняется при $u$ из (48). В качестве примера можно привести функции, которые ведут себя как $\exp (\lambda x), \lambda>0$, на некоторых интервалах значений аргумента $x$. Заметим, впрочем, что утверждение следствия 7 при $H(x) \equiv \exp (\lambda x)$ легко выводится из теоремы 2.

Пример функций $H$, удовлетворяющих условию $G(\cdot) \in \mathscr{G}(u)$ с $u$ из (48), содержится в работе автора [99] (см. раздел 5, следствия 5 и 6). Пусть $H-$ такая неотрицательная функция, что $H(0)=0$, функция $H(x) / x^{2}$ не убывает, а функция $H(x) / x^{\lambda}$ не возрастает при некотором $\lambda>2$. Тогда при доказательстве следствия 5 в [99] было показано, что $G(\cdot) \in \mathscr{G}(u)$ при $u=H^{-1}\left(e^{\lambda} L_{H}\right)$. В частности, можно рассмотреть $H(x)=x^{\gamma}, \gamma>2$. Таким образом, следствие 7 позволяет устранить логарифмические ограничения на значения $z$ из формулировок следствий 5 и 6 в случае одинаково распределенных слагаемых. Заметим, что в недавней совместной работе $\Phi$. Гётце и автора [42] содержится даже более сильное утверждение в случае $H(x)=x^{\gamma}, \gamma>2$. Показано, что существует конструкция, для которой $\mathrm{E}\left(\Delta_{n}(X, Y)\right)^{\gamma} \leqslant a(d, \gamma) n \mathrm{E}\|X\|^{\gamma}$ (см. теорему 11).

Еще один пример дает функция $H(x)=\exp \left(\lambda x^{\beta}\right), 0<\beta<1$. В этом случае $G(\cdot) \in \mathscr{G}(u)$ при $u=(1-\beta)^{-1 / \beta} H^{-1}\left(L_{H}\right)$. Ясно, что список примеров может быть продолжен.

\section{7. Точность аппроксимации в задаче (B) для сумм неодинаково распределенных векторов с конечными степенными моментами}

В дальнейшем мы будем писать $A \ll_{t} B$, если существует такая зависящая только от $t$ положительная величина $c_{t}$, что $A \leqslant c_{t} B$. Мы будем также писать $A \asymp_{t} B$, если $A \ll_{t} B \ll_{t} A$. Отсутствие нижнего индекса означает, разумеется, что соответствующие постоянные являются абсолютными.

В этом разделе приведены результаты, опубликованные в совместной работе $\Phi$. Гётце и автора [41] (см. теорему 10). Они относятся к задаче (В) для сумм 
неодинаково распределенных векторов с конечными моментами $\mathrm{E}\left\|X_{j}\right\|^{\gamma}<\infty$, где $\gamma>2$.

В теореме 10 предпринята новая попытка получить многомерные обобщения результатов Саханенко [73] для случая $H(x)=x^{\gamma}$. Первая из них была осуществлена в работе автора [99] (см. следствие 6), но соответствующее неравенство было получено не для всех возможных значений аргумента $z$.

Теорема 10. Предположим, что $\alpha>0 u X_{1}, \ldots, X_{n}$ - независимые $d$-мерные случайные векторы с $\mathrm{E} X_{j}=0, j=1, \ldots, n$. Пусть $\gamma \geqslant 2$ и величина $L_{\gamma}$ определена равенством (40). Предположим, что выполнено условие $\mathbf{B}_{s}\left(w^{2}, C_{1} w^{2}\right)$, где

$$
w=\frac{C_{2} L_{\gamma}^{1 / \gamma}}{\log ^{*} s}
$$

с некоторыми положительными постоянными $C_{1} u C_{2}$. Предположим, что величинь

$$
\lambda_{k, \gamma}=\sum_{j=m_{k-1}+1}^{m_{k}} \mathrm{E}\left\|X_{j}\right\|^{\gamma}, \quad k=1, \ldots, s,
$$

для некоторого $0<\varepsilon<1$ удовлетворяют неравенству

$$
C_{3} d^{\gamma / 2} s^{\varepsilon}\left(\log ^{*} s\right)^{\gamma+3} \max _{1 \leqslant k \leqslant s} \lambda_{k, \gamma} \leqslant L_{\gamma}
$$

с положительной постоянной $C_{3}$. Тогда существует такое построение, что для любого $z>a_{16} \varepsilon^{-1} L_{\gamma}^{1 / \gamma} d^{21 / 2+\alpha} \log ^{*} d$ и любого $b \geqslant 1$

$$
\begin{gathered}
\begin{aligned}
& \mathrm{P}\left\{\Delta_{n}(X, Y) \geqslant 9 z\right\} \leqslant 1 d^{1+\gamma b / 2}(2 b)^{b(\gamma-1)} z^{-\gamma b} \sum_{k=1}^{s} \lambda_{k, \gamma}^{b}+\exp \left(-\frac{a_{17} z^{2}}{d L_{\gamma}^{2 / \gamma}}\right) \\
&+\exp \left(-\frac{a_{18} \varepsilon z \log ^{*} s}{L_{\gamma}^{1 / \gamma} d^{15 / 2} \log ^{*} d}\right)+5 d \sum_{j=1}^{n} \mathrm{P}\left\{\left\|X_{j}\right\| \geqslant \frac{z}{2 b \sqrt{d}}\right\} \\
& \mathrm{P}\left\{\Delta_{n}(X, Y) \geqslant 9 z\right\} \leqslant d^{1+\gamma / 2}\left(a_{19} b^{4} \varepsilon^{-1}\right)^{\gamma b}\left(L_{\gamma} z^{-\gamma}\right)^{b}
\end{aligned} \\
\quad+5 d \sum_{j=1}^{n} \mathrm{P}\left\{\left\|X_{j}\right\| \geqslant \frac{z}{2 b \sqrt{d}}\right\} \\
\mathrm{P}\left\{\Delta_{n}(X, Y) \geqslant z\right\} \leqslant a_{20} d^{1+\gamma / 2} L_{\gamma}(\varepsilon z)^{-\gamma}
\end{gathered}
$$

$u$

$$
\mathrm{E}\left(\Delta_{n}(X, Y)\right)^{\gamma} \leqslant a_{21}\left(\varepsilon^{-1} d^{21 / 2+\alpha} \log ^{*} d\right)^{\gamma} L_{\gamma},
$$

где $a_{16}, \ldots, a_{21}$ - некоторые положительные величинь, зависящие только от $\alpha$, $\gamma, C_{1}, C_{2} u C_{3}$.

Заметим, что при $z \leqslant a_{16} \varepsilon^{-1} L_{\gamma}^{1 / \gamma} d^{21 / 2+\alpha} \log ^{*} d$ в условиях теоремы 10 справедливы неравенства

$$
\mathrm{P}\left\{\Delta_{n}(X, Y) \geqslant z\right\} \leqslant 1 \leqslant\left(a_{16} \varepsilon^{-1} d^{21 / 2+\alpha} \log ^{*} d\right)^{\gamma} L_{\gamma} z^{-\gamma}
$$


С другой стороны, из неравенства (55) следует, что

$$
\mathrm{P}\left\{\Delta_{n}(X, Y) \geqslant z\right\} \leqslant a_{21}\left(\varepsilon^{-1} d^{21 / 2+\alpha} \log ^{*} d\right)^{\gamma} L_{\gamma} z^{-\gamma}
$$

для всех $z>0$. Таким образом, в теореме 10 неравенство типа (29) установлено, на самом деле, для всех возможных значений z. Невозможно, впрочем, вывести из (55) неравенство (54), которое точнее в смысле зависимости констант от размерности $d$. Заметим, что в этой теореме мы не пытаемся оптимизировать зависимость констант от размерности. Важно, что эта зависимость имеет степенной характер. Ясно также, что (55) не вытекает из (56) или из (54). Кроме того, иногда неравенство (53) оказывается сильнее, чем (54) (например, если последняя сумма в (53) равна нулю). Неравенство (52) точнее, чем (53) и (54). С другой стороны, неравенства (53) и (54) формулируются проще, чем (52).

Условие (51) довольно громоздко, но оно тривиально выполняется, если векторы $X_{1}, \ldots, X_{n}$ имеют одно и то же распределение $F$ и $n$ достаточно велико (см. теорему 11). Действительно, ясно, что в этом случае $m_{k} \asymp_{F} k n^{2 / \gamma} /\left(\log ^{*} n\right)^{2}$, $\lambda_{k, \gamma} \asymp_{F} n^{2 / \gamma} /\left(\log ^{*} n\right)^{2}, k=1, \ldots, s, s \asymp_{F} n^{1-2 / \gamma}\left(\log ^{*} n\right)^{2}$ и условие (51) превращается в

$$
C_{3} d^{\gamma / 2} n^{\varepsilon(1-2 / \gamma)}\left(\log ^{*} n\right)^{\gamma+1+2 \varepsilon} \leqslant A n^{1-2 / \gamma}
$$

с некоторым $A$, не зависящим от $n$. Кроме того, в общем случае

$$
L_{\gamma}=\sum_{k=1}^{s} \lambda_{k, \gamma}
$$

и (51) должно быть выполнено, если величины $\lambda_{k, \gamma}$ ведут себя регулярно.

Пусть $2 \leqslant \beta<\gamma$. Тогда в условиях теоремы 10

$$
\left(\mathrm{E}\left(\Delta_{n}(X, Y)\right)^{\beta}\right)^{1 / \beta} \leqslant\left(\mathrm{E}\left(\Delta_{n}(X, Y)\right)^{\gamma}\right)^{1 / \gamma} \leqslant a_{21}^{1 / \gamma} \varepsilon^{-1} d^{21 / 2+\alpha} \log ^{*} d \cdot L_{\gamma}^{1 / \gamma} .
$$

Эта оценка может быть лучше оценки, получающейся из теоремы 10 с $\gamma=\beta$. Например, в случае одинаково распределенных векторов справедливо соотношение $L_{\gamma}^{1 / \gamma}=\left(n \mathrm{E}\left\|X_{1}\right\|^{\gamma}\right)^{1 / \gamma} \asymp_{F} n^{1 / \gamma}$, тогда как $L_{\beta}^{1 / \beta}=\left(n \mathrm{E}\left\|X_{1}\right\|^{\beta}\right)^{1 / \beta} \asymp_{F} n^{1 / \beta}$. В общем случае, для фиксированного $\beta$, следует пытаться минимизировать $L_{\gamma}^{1 / \gamma}$, $\gamma \geqslant \beta$. Заметим, впрочем, что условия $\mathbf{B}_{s}\left(w^{2}, C_{1} w^{2}\right)$ и (51) различны для различных $\gamma$.

Доказательство теоремы 10 представляет собой видоизмененный вариант соответствующих доказательств из работ Саханенко [73] и автора [99]. Главное отличие в том, что мы урезаем не исходные слагаемые $X_{j}$, а суммы $\zeta_{k}$ по блокам слагаемых $X_{j}$. Затем устанавливается, что распределения урезанных векторов принадлежат классу $\mathscr{A}_{d}(\tau)$ с $\tau$, существенно меньшим, чем уровень урезания $u$. Аналогичная идея была использована Айнмалем [31]. Полное доказательство Саханенко [73] пока обобщить не удалось из-за технических трудностей, возникающих в многомерном случае.

Для удобства сравнений приведем несколько оценок, которые справедливы при любом способе задания случайных векторов $X_{1}, \ldots, X_{n}$ и $Y_{1}, \ldots, Y_{n}$ на 
одном вероятностном пространстве. Все эти оценки вытекают из простого и грубого неравенства

$$
\Delta_{n}(X, Y) \leqslant \max _{1 \leqslant s \leqslant n}\left\|\sum_{j=1}^{s} X_{j}\right\|+\max _{1 \leqslant s \leqslant n}\left\|\sum_{j=1}^{s} Y_{j}\right\|
$$

и последующего оценивания слагаемых в его правой части.

ПреДЛОЖЕНИЕ 1. Пусть $X_{1}, \ldots, X_{n}$ - независимые одинаково распределенные случайные векторы, которые имеют нулевые средние и принимают значения в сепарабельном гилъбертовом пространстве Н. Тогда для любого построения справедливо неравенство

$$
\mathrm{E}\left(\Delta_{n}(X, Y)\right)^{\gamma} \ll_{\gamma} n \mathrm{E}\left\|X_{j}\right\|^{\gamma}+\left(n \mathrm{E}\left\|X_{j}\right\|^{2}\right)^{\gamma / 2} \quad n p u \gamma \geqslant 2 .
$$

Этот факт вытекает из (57) и многомерного варианта неравенства Розенталя, который легко следует из одного результата де Акоста [22] и того, что Н является пространством типа 2. Кроме того, используется один результат Монтгомери-Смита [58], представляющего собой частный случай теоремы 1.1.5 из монографии де ля Пенья и Жине [23]. Из этого результата следует, что

$$
\mathrm{E} \max _{1 \leqslant s \leqslant n}\left\|\sum_{j=1}^{s} X_{j}\right\|^{\gamma} \ll_{\gamma} \mathrm{E}\left\|\sum_{j=1}^{n} X_{j}\right\|^{\gamma}, \quad \gamma>0
$$

в случае независимых одинаково распределенных векторов $X_{1}, \ldots, X_{n}$.

Второе слагаемое в правой части неравенства (58) предложения 1 растет быстрее, чем первое, при $n \rightarrow \infty$. Теоремы 6 и 11 показывают, что в конечномерной ситуации этот рост соответствует росту сумм гауссовских аппроксимирующих векторов.

ПреДЛОжЕНИЕ 2. Пусть $\gamma \geqslant 2, b \geqslant 1, a X_{1}, \ldots, X_{n}$-независимые $d$-мерные случайные векторы с нулевыми средними. Тогда для любого построения справедливо неравенство

$$
\begin{aligned}
\mathrm{P}\left\{\Delta_{n}(X, Y) \geqslant 6 \sqrt{d} x\right\} \leqslant 6 & \exp \left(-\frac{a_{22} x^{2}}{\sigma^{2}}\right)+2(2 b)^{b(\gamma-1)}\left(\sum_{j=1}^{n} \frac{\mathrm{E}\left\|X_{j}\right\|^{\gamma}}{x^{\gamma}}\right)^{b} \\
+ & \sum_{j=1}^{n} \mathrm{P}\left\{\left\|X_{j}\right\| \geqslant \frac{x}{2 b}\right\}, \quad x>0,
\end{aligned}
$$

где $\sigma^{2}$ - максимальное собственное значение оператора $\operatorname{cov}\left(\sum_{j=1}^{n} X_{j}\right)$, а $a_{22}-$ положительная величина, зависящая только от $\gamma$. В частности, при $b=1$ для любого построения выполнено неравенство

$$
\mathrm{P}\left\{\Delta_{n}(X, Y) \geqslant 6 \sqrt{d} x\right\} \leqslant 6 \exp \left(-\frac{a_{22} x^{2}}{\sigma^{2}}\right)+2^{\gamma+1} \sum_{j=1}^{n} \frac{\mathrm{E}\left\|X_{j}\right\|^{\gamma}}{x^{\gamma}}, \quad x>0 .
$$


Предложение 2 легко следует из (57) и покоординатного применения основного результата Д. Х. Фука и С. В. Нагаева [38], см. также следствие 1.7 в [59]. Используется также вариант неравенства Оттавиани

$$
\mathrm{P}\left\{\max _{1 \leqslant k \leqslant n}\left\|X_{1}+\cdots+X_{k}\right\| \geqslant 3 t\right\} \leqslant 3 \max _{1 \leqslant k \leqslant n} \mathrm{P}\left\{\left\|X_{1}+\cdots+X_{k}\right\| \geqslant t\right\}, \quad t \geqslant 0
$$

(см. предложение 1.1.2 из монографии де ля Пенья и Жине [23]). В виде (62) это неравенство можно найти в [51] и (с 4 вместо 3 (два раза)) в [37].

Из условий $\mathbf{B}_{s}\left(r^{2} L_{\gamma}^{2 / \gamma}, C r^{2} L_{\gamma}^{2 / \gamma}\right)$ и $\mathbf{B}_{s}\left(w^{2}, C_{1} w^{2}\right)$ следует, что минимальное собственное значение оператора $\operatorname{cov}\left(\sum_{j=1}^{n} X_{j}\right)$ ограничено снизу соответственно величинами $r^{2} L_{\gamma}^{2 / \gamma}$ и $w^{2}$. Предложение 2 показывает, что если в условиях теоремы 10 собственные значения $\operatorname{cov}\left(\sum_{j=1}^{n} X_{j}\right)$ невелики (меньше, чем $C_{4} L_{\gamma}^{2 / \gamma}$ с некоторой положительной постоянной $C_{4}$ ), то требуемые неравенства справедливы для любого построения случайных векторов $\left\{X_{j}\right\}$ и $\left\{Y_{j}\right\}$ на одном и том же вероятностном пространстве. В частности,

$$
\mathrm{E}\left(\Delta_{n}(X, Y)\right)^{\gamma} \leqslant a_{23} d^{1+\gamma / 2} L_{\gamma}
$$

где $a_{23}$ - положительная величина, зависящая только от $\gamma$ и $C_{4}$.

Подчеркнем, что при сильной аппроксимации основной целью является доказательство существования таких способов задания векторов $X_{1}, \ldots, X_{n}$ и $Y_{1}, \ldots, Y_{n}$ на одном вероятностном пространстве, чтобы имели место лучшие оценки (по сравнению с тривиальными неравенствами из приведенных выше предложений).

\section{8. Точность аппроксимации в задаче (В) для сумм одинаково распределенных векторов с конечными степенными моментами}

В этом разделе мы покажем, какие конечномерные следствия вытекают из теоремы 10 в случае одинаково распределенных слагаемых (см. теоремы 11 и 12$)$.

В формулировках следующих ниже результатов разделов 8-11 будет участвовать случайный вектор $X=\left(\mathscr{X}_{1}, \mathscr{X}_{2}, \ldots\right)$, принимающий значения в конечномерном или бесконечномерном сепарабельном гильбертовом пространстве. Независимые копии вектора $X$ требуется построить на одном вероятностном пространстве с последовательностью независимых гауссовских случайных векторов. Не нарушая общности, мы будем предполагать, что координаты вектора $X$ некоррелированы, причем

$$
\sigma_{1}^{2} \geqslant \sigma_{2}^{2} \geqslant \cdots \geqslant \sigma_{m}^{2} \geqslant \cdots, \quad \text { где } \sigma_{m}^{2}=\mathrm{E} \mathscr{X}_{m}^{2}, \quad m=1,2, \ldots
$$

Координаты вектора $X$ обозначаются через $\mathscr{X}_{m}$, а не $X_{m}$, чтобы отличить их от независимых копий вектора $X$, участвующих в построении. 
Как правило, мы будем рассматривать ситуацию, в которой

$$
\sigma_{d}^{2}>0, \quad \mathrm{E} X=0 \quad \text { и } \quad \mathrm{E}\|X\|^{\gamma}<\infty
$$

при некотором $\gamma \geqslant 2$. Кроме того, в формулировках будет присутствовать неслучайный параметр $\psi$, удовлетворяющий неравенству

$$
\frac{21}{2}<\psi \leqslant 11
$$

В дальнейшем многие константы будут зависеть от $\psi$. Чтобы избавиться от этого усложнения, можно, например, взять $\psi=11$.

ТеОрема 11. Пусть $X-\mathbb{R}^{d}$-значный случайный вектор и выполнены условия (65) и (66). Тогда существует такое построение, что

$$
\mathrm{E}\left(\Delta_{n}(X, Y)\right)^{\gamma} \ll_{\gamma, \psi} A\left(\frac{\sigma_{1}}{\sigma_{d}}\right)^{\gamma} n \mathrm{E}\|X\|^{\gamma} \quad \text { при всех } n,
$$

где

$$
A=A(\gamma, \psi, d)=\max \left\{d^{\psi \gamma}, d^{\gamma(\gamma+2) / 4}\left(\log ^{*} d\right)^{\gamma(\gamma+1) / 2}\right\} .
$$

Ясно, что теорема 11 представляет собой многомерный вариант теоремы 6 для одинаково распределенных слагаемых.

Применяя неравенство Чебышёва, мы видим, что в условиях теоремы 11

$$
\mathrm{P}\left\{\Delta_{n}(X, Y) \geqslant x\right\} \ll_{\gamma, d}\left(\frac{\sigma_{1}}{\sigma_{d}}\right)^{\gamma} \frac{n \mathrm{E}\|X\|^{\gamma}}{x^{\gamma}}
$$

для всех $x>0$ и всех $n=1,2, \ldots$ Я Ясно, что утверждение теоремы 11 сильнее, чем (69). Построение, для которого (69) справедливо при $d=1$ при фиксированных $n$ и $x=O(\sqrt{n} \log n)$ с константами, зависящими только от $\gamma$ и $\mathscr{L}(X)$, было предложено KMT [50], см. также работы А. А. Боровкова [9] и П. Майора [53] в случае $2<\gamma \leqslant 3$. Затем А. И. Саханенко [73] доказал теорему 6, которая обеспечивает справедливость (69) для всех $x$ на одном и том же вероятностном пространстве. У. Айнмаль [31] получил многомерный вариант результата KMT без ограничений на значения аргумента $x$.

В следующем разделе мы рассмотрим бесконечномерные результаты, для получения которых была использована несколько иная конечномерная формулировка. Следующее утверждение было доказано в работе [42] в процессе доказательства теоремы 11.

ТеОРема 12. Пусть $X-\mathbb{R}^{d}$-значный случайный вектор и выполнены условия (65) и (66). Тогда существует такая зависящая только от $\gamma$ положительная величина $C(\gamma)$, что при каждом фиксированном натуральном $n$, удовлетворяющем неравенству

$$
C(\gamma) d^{\gamma / 2}\left(\log ^{*} d\right)^{\gamma+1}\left(\mathrm{E}\left\|\mathbb{D}^{-1 / 2} X\right\|^{\gamma}\right)^{2 / \gamma} \leqslant n^{1-2 / \gamma},
$$

существует такое построение, что

$$
\mathrm{E}\left(\Delta_{n}\left(\mathbb{D}^{-1 / 2} X, \mathbb{D}^{-1 / 2} Y\right)\right)^{\gamma} \ll_{\gamma, \psi} d^{\psi \gamma} n \mathrm{E}\left\|\mathbb{D}^{-1 / 2} X\right\|^{\gamma} .
$$


В работе [42] в формулировках теорем 11 и 12 при $d^{\psi \gamma}$ присутствует дополнительный логарифмический множитель $\left(\log ^{*} d\right)^{2 \gamma}$. От него можно легко избавиться, учитывая, что для констант в неравенствах (67) и (71) допускается зависимость от $\psi$, удовлетворяющего неравенству (66).

Если условие (70) не выполнено, то оценка в теореме 11 получается не за счет удачной аппроксимации, а за счет того, что величина $\mathrm{E}\left(\Delta_{n}(X, Y)\right)^{\gamma}$ оценивается сверху с помощью предложения 1. Так что наличие в формулировке теоремы 12 условия (70) не приводит к потере информации о близости распределений по сравнению с теоремой 11.

\section{9. Бесконечномерный случай}

В этом разделе мы покажем, какие бесконечномерные следствия вытекают из конечномерной теоремы 12. В работе [43] из этой теоремы 12 были выведены содержательные оценки точности сильной гауссовской аппроксимации сумм независимых одинаково распределенных гильбертовозначных случайных векторов $X_{j}$ с $\mathscr{L}\left(X_{j}\right)=\mathscr{L}(X)$, имеющих конечные моменты $\mathrm{E}\|X\|^{\gamma}, \gamma>2$ (см. теоремы 13 и 14 ниже). Оказалось, что точность аппроксимации существенно зависит от скорости убывания последовательности собственных чисел ковариационного оператора слагаемых.

Теоремы 13 и 14 можно рассматривать как бесконечномерные аналоги теоремы 6 в случае независимых одинаково распределенных слагаемых.

В разделах 9-11 символ Н обозначает сепарабельное гильбертово пространство, состоящее из вещественных последовательностей $x=\left(x_{1}, x_{2}, \ldots\right)$, для которых $\|x\|^{2}=x_{1}^{2}+x_{2}^{2}+\cdots<\infty$. Мы будем также обозначать $x_{(d)}=$ $\left(x_{1}, x_{2}, \ldots, x_{d}\right) \in \mathbb{R}^{d}$ и $x_{[d]}=\left(0,0, \ldots, 0, x_{d}, x_{d+1}, \ldots\right) \in \mathbf{H}$.

Обозначим

$$
\mathbb{D}=\operatorname{cov} X, \quad \mathbb{D}_{d}=\operatorname{cov} X_{(d)}, \quad B_{d}^{2}=\sum_{m=d+1}^{\infty} \sigma_{m}^{2}=\mathrm{E}\left\|X_{[d]}\right\|^{2} .
$$

В частности,

$$
B_{0}^{2}=\sum_{m=1}^{\infty} \sigma_{m}^{2}=\mathrm{E}\|X\|^{2} .
$$

Следующие теоремы 13 и 14 были доказаны в работе [43].

Теорема 13. Пусть X - Н-значный случайный вектор и выполнены условия (65) и (66) при некотором $\gamma>2$. Тогда существует такая зависящая только от $\gamma$ положительная величина $C(\gamma)$, что при любых фиксированных натуральных $n$ и d, удовлетворяющих неравенству

$$
C(\gamma) d^{\gamma / 2}\left(\log ^{*} d\right)^{\gamma+1}\left(\mathrm{E}\left\|\mathbb{D}_{d}^{-1 / 2} X_{(d)}\right\|^{\gamma}\right)^{2 / \gamma} \leqslant n^{1-2 / \gamma}
$$

существует такое построение, что

$$
\mathrm{E}\left(\Delta_{n}(X, Y)\right)^{\gamma} \ll_{\gamma, \psi} d^{\psi \gamma} n \sigma_{1}^{\gamma} \mathrm{E}\left\|\mathbb{D}_{d}^{-1 / 2} X_{(d)}\right\|^{\gamma}+n \mathrm{E}\left\|X_{[d]}\right\|^{\gamma}+\left(n B_{d}^{2}\right)^{\gamma / 2} .
$$




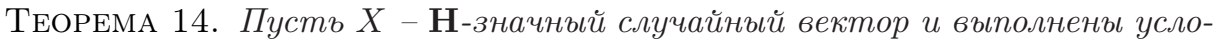
вия (65) и (66) при некотором $\gamma>2$. Тогда существует такая зависящая только от $\gamma$ положительная величина $C(\gamma)$, что при любых фиксированных натуральных $n$ u d, удовлетворяющих неравенству

$$
C(\gamma) d^{\gamma / 2}\left(\log ^{*} d\right)^{\gamma+1}\left(\mathrm{E}\|X\|^{\gamma}\right)^{2 / \gamma} \leqslant n^{1-2 / \gamma} \sigma_{d}^{2},
$$

существует такое построение, что

$$
\mathrm{E}\left(\Delta_{n}(X, Y)\right)^{\gamma} \ll_{\gamma, \psi} d^{\psi \gamma}\left(\frac{\sigma_{1}}{\sigma_{d}}\right)^{\gamma} n \mathrm{E}\|X\|^{\gamma}+\left(n B_{d}^{2}\right)^{\gamma / 2} .
$$

Теоремы 13 и 14 дают возможность получать содержательные бесконечномерные оценки за счет подходящего выбора размерности $d$, при котором слагаемые в правых частях неравенств (75) или (77) имеют примерно одинаковый порядок по $n$. Теорема 14 является элементарным следствием теоремы 13 и неравенства

$$
\mathrm{E}\left\|\mathbb{D}_{d}^{-1 / 2} X_{(d)}\right\|^{\gamma} \leqslant \sigma_{d}^{-\gamma} \mathrm{E}\left\|X_{(d)}\right\|^{\gamma} \leqslant \sigma_{d}^{-\gamma} \mathrm{E}\|X\|^{\gamma} .
$$

Вообще говоря, теорема 13 точнее теоремы 14. Для многих распределений с регулярным поведением моментов справедливо соотношение

$$
K=\sup _{1 \leqslant d<\infty} d^{-\gamma / 2} \mathrm{E}\left\|\mathbb{D}_{d}^{-1 / 2} X_{(d)}\right\|^{\gamma}<\infty
$$

что может дать существенное уточнение порядка оценок. Например, если вектор $X$ имеет независимые координаты $\mathscr{X}_{m}$, то

$$
\mathrm{E}\left\|\mathbb{D}_{d}^{-1 / 2} X_{(d)}\right\|^{\gamma}=\mathrm{E}\left(\sum_{m=1}^{d} \frac{\mathscr{X}_{m}^{2}}{\sigma_{m}^{2}}\right)^{\gamma / 2} \ll_{\gamma} d^{\gamma / 2}+\sum_{m=1}^{d} \sigma_{m}^{-\gamma} \mathrm{E}\left|\mathscr{X}_{m}\right|^{\gamma} .
$$

Следовательно, $K<\infty$, если последовательность моментов $\sigma_{m}^{-\gamma} \mathrm{E}\left|\mathscr{X}_{m}\right|^{\gamma}$ ограничена или растет не быстрее, чем $O\left(m^{(\gamma-2) / 2}\right)$. Заметим, что, в силу неравенства Ляпунова, $\mathrm{E}\left\|\mathbb{D}_{d}^{-1 / 2} X_{(d)}\right\|^{\gamma} \geqslant d^{\gamma / 2}$.

С другой стороны, в общем случае применение неравенства (78) может не приводить к потере точности оценки, а формулировка теоремы 14 проще формулировки теоремы 13. В ней участвуют только момент $\mathrm{E}\|X\|^{\gamma}$ и собственные числа ковариационного оператора $\mathbb{D}$ вектора $X$. Возможна промежуточная ситуация, когда неравенство (79) не выполняется, но утверждение теоремы 13 все еще сильнее утверждения теоремы 14.

Доказательство теорем 13 и 14 основано на методе конечномерной аппроксимации, родственном методу, применяемому при оценивании точности аппроксимации в центральной предельной теореме в бесконечномерных пространствах (см., например, обзор [2]).

Ранее оценки точности сильной аппроксимации в бесконечномерных пространствах были получены, например, в работах [11]-[13], [74] и [76].

Наиболее близок к тематике данного раздела следующий бесконечномерный результат А. И. Саханенко [76]. 
Tеорема 15. Предположим, что $X$ есть Н-значный случайный вектор $c$ $\mathrm{E} X=0 u \mathrm{E}\|X\|^{\gamma}<\infty$ при некотором $\gamma, 2 \leqslant \gamma \leqslant 3$. Тогда для любого фиксированного $x>0$ существует такое построение, что

$$
\mathrm{P}\left\{\Delta_{n}^{\infty}(X, Y) \geqslant x\right\} \ll \frac{n \mathrm{E}\|X\|^{\gamma}}{x^{\gamma}} \text { nрu всех } n
$$

¿əe

$$
\Delta_{n}^{\infty}(X, Y)=\max _{1 \leqslant s \leqslant n}\left\|\sum_{j=1}^{s} X_{j}-\sum_{j=1}^{s} Y_{j}\right\|_{\infty}
$$

$u\|x\|_{\infty}=\max _{j}\left|x_{j}\right|$ для $x \in \mathbf{H}$.

Теорема 15 сформулирована при фиксированном $x$. Это означает, что вероятностное пространство зависит от этого $x$. Кроме того, в формулировке теоремы 15 вместо $\Delta_{n}(X, Y)$ фигурирует величина $\Delta_{n}^{\infty}(X, Y)$, которая, вообще говоря, может оказаться существенно меньше, чем $\Delta_{n}(X, Y)$. С другой стороны, неравенство (81) выглядит почти так же, как неравенство (69) при $2 \leqslant \gamma \leqslant 3$. Следует также отметить, что А. И. Саханенко [76] получил существенно более общие результаты по сравнению с теоремой 15. Они доказаны для разнораспределенных зависимых слагаемых, образующих, например, бесконечномерные мартингалы.

Теорема 15 может быть полезной и в конечномерной ситуации (см., например, [5]), поскольку оценка (81) не вырождается, когда минимальное собственное число ковариационного оператора вектора $X$ стремится к нулю.

Следующая теорема 16 была доказана в работе [43]. Она дает оценку снизу в условиях теорем 13 и 14.

ТеОРема 16. Пусть положителъные числа $\sigma_{m}^{2}, m=1,2, \ldots$, удовлетворяют соотношениям

$$
\sigma_{1}^{2} \geqslant \sigma_{2}^{2} \geqslant \cdots \geqslant \sigma_{m}^{2} \geqslant \cdots \quad u \sum_{m=1}^{\infty} \sigma_{m}^{2}<\infty .
$$

Пусть $n$ - фиксированное натуральное число, а $\lambda>0$, причем $\sigma_{1}^{2} \leqslant \lambda^{2}$. Обозначим

$$
k=\min \left\{m: n \sigma_{m}^{2}<\lambda^{2}\right\}-1 .
$$

Тогда существует такой случайный вектор $X=\left(\mathscr{X}_{1}, \mathscr{X}_{2}, \ldots\right)$, принимающий значения в $\mathbf{H}$ и удовлетворяющий соотношениям (64), (72) и (73), что $\mathrm{E}\|X\|^{\gamma}<\infty$ при всех $\gamma \geqslant 0$ и для любого построения справедлива оченка снизу

$$
\mathrm{E}\left(\Delta_{n}(X, Y)\right)^{\gamma} \gg_{\gamma} \mathrm{E}\left(\Delta_{n}\left(X_{(k)}, Y_{(k)}\right)\right)^{\gamma}+\left(n B_{k}^{2}\right)^{\gamma / 2} .
$$

При этом первое слагаемое в правой части неравенства (85) считается равнъцм нулю, если $k=0$.

При доказательстве теоремы 16 в качестве $\mathscr{X}_{m}$ (координат вектора $X$ ) выбираются независимые случайные величины, принимающие значения $-\lambda, 0$ и $\lambda$ с вероятностями

$$
\mathrm{P}\left\{\mathscr{X}_{m}= \pm \lambda\right\}=\frac{\sigma_{m}^{2}}{2 \lambda^{2}}, \quad \mathrm{P}\left\{\mathscr{X}_{m}=0\right\}=1-\frac{\sigma_{m}^{2}}{\lambda^{2}}, \quad m=1,2, \ldots
$$

Нетрудно показать, что $\mathrm{E}\|X\|^{\gamma}<\infty$ при всех $\gamma \geqslant 0$. 
Оценивание снизу величины $\mathrm{E}\left(\Delta_{n}\left(X_{(k)}, Y_{(k)}\right)\right)^{\gamma}$ представляет собой отдельную задачу. Впрочем, для вектора $X$ из доказательства теоремы 16 справедлива грубая оценка $\mathrm{E}\left(\Delta_{n}\left(X_{(k)}, Y_{(k)}\right)\right)^{\gamma} \gg_{\gamma}\left(\lambda^{2} k\right)^{\gamma / 2}$, основанная на решетчатости распределения этого вектора.

Наличие в правой части оценки снизу (85) величины $\left(n B_{k}^{2}\right)^{\gamma / 2}$ свидетельствует о естественности появления слагаемого $\left(n B_{d}^{2}\right)^{\gamma / 2}$ в оценках сверху $(75)$ и $(77)$.

В разделе 10 мы рассмотрим примеры 1-4, показывающие, в частности, что для многих распределений теорема 14 дает оценки, которые лучше по порядку, чем оценка теоремы 15. Кроме того, в примере 5 и в разделе 11 мы покажем, что если последовательность собственных чисел $\sigma_{m}^{2}$ убывает медленно, то теоремы 13 и 14 дают правильные по порядку оценки.

Теоремы 12-14 сформулированы при фиксированном $n$, а оценка в теореме 11 справедлива при всех $n$ одновременно на одном и том же вероятностном пространстве. Первоначально в работе [42] она была доказана при фиксированном $n$. Чтобы получить построение, дающее результат для всех $n$, достаточно использовать независимые построения с фиксированными $n \asymp 2^{m}$, $m=1,2, \ldots$. То же самое будет справедливо и для порядка зависимости от $n$ оценок, выписанных при рассмотрении примеров 1-5. Действуя аналогичным образом, не составляет труда перенести оценки, полученные на вероятностных пространствах, зависящих от $n$, на вероятностное пространство, на котором оценки соответствующего порядка будут верны для всех $n$.

\section{0. Бесконечномерные примеры}

В примерах 1-5 мы сравним оценки, которые дает теорема 13 при выполнении условия (79), с оценками, вытекающими из теоремы 14 для конкретных последовательностей собственных чисел ковариационного оператора вектора $X$.

ПримеР 1. Пусть $\sigma_{m}^{2}=\exp \left\{-\alpha m^{\beta}\right\}, m=1,2, \ldots$, где $\alpha, \beta>0$. Будем считать, что $n$ настолько велико, что

$$
d=\max \left\{m: \frac{n^{2 / \gamma}\left(\log ^{*} n\right)^{2 \psi / \beta}}{\sigma_{m}^{2}}<n \sigma_{m}^{2}\right\} \geqslant 1 .
$$

Тогда при достаточно большом $n$ справедливо утверждение теоремы 14 с оценкой

$$
\mathrm{E}\left(\Delta_{n}(X, Y)\right)^{\gamma} \ll_{\alpha, \beta, \gamma, \psi} n^{(2+\gamma) / 4}\left(\log ^{*} n\right)^{\psi \gamma / 2 \beta} \mathrm{E}\|X\|^{\gamma} .
$$

Правая часть неравенства (88) растет медленнее, чем $n^{\gamma / 2}$ (порядок тривиальной оценки, вытекающей из предложения 1). Поэтому неравенство (88) представляет собой содержательную оценку точности аппроксимации в бесконечномерном принципе инвариантности. В частности, при $\gamma>3$ из (88) и неравенства $\mathrm{E} \Delta^{3} \leqslant\left(\mathrm{E} \Delta^{\gamma}\right)^{3 / \gamma}$ следует, что

$$
\mathrm{E}\left(\Delta_{n}(X, Y)\right)^{3} \ll_{\alpha, \beta, \gamma, \psi} n^{3(2+\gamma) /(4 \gamma)}\left(\log ^{*} n\right)^{3 \psi /(2 \beta)}\left(\mathrm{E}\|X\|^{\gamma}\right)^{3 / \gamma}
$$

При $\gamma>6$ порядок неравенства (89) по $n$ лучше, чем порядок оценки (81). 
ПримеР 2. Предположим теперь, что в условиях примера 1 выполнено соотношение (79). Пусть $n$ настолько велико, что

$$
d=\min \left\{m: n B_{m}^{2}<1\right\} \geqslant 1
$$

Тогда при достаточно большом $n$ справедливо утверждение теоремы 13 с оценкой

$$
\mathrm{E}\left(\Delta_{n}(X, Y)\right)^{\gamma} \ll_{\alpha, \beta, \gamma, \psi, K} n\left(\log ^{*} n\right)^{(2 \psi+1) \gamma /(2 \beta)} \mathrm{E}\|X\|^{\gamma},
$$

которая значительно сильнее неравенства (88) и близка к конечномерной оценке (67) теоремы 11.

ПримеР 3. Пусть $\sigma_{m}^{2}=m^{-b}, m=1,2, \ldots$, где $b>1$. Выберем

$$
d=\max \left\{m: \frac{n^{2 / \gamma} m^{2 \psi}}{\sigma_{m}^{2}}<n m \sigma_{m}^{2}\right\} \geqslant 1 .
$$

Если же условие (76) не выполнено для $d$, определенного с помощью равенства (92), следует уменьшить $d$, выбирая его с помощью соотношения

$$
d=\max \left\{m: C(\gamma) m^{\gamma / 2}\left(\log ^{*} m\right)^{\gamma+1}\left(\mathrm{E}\|X\|^{\gamma}\right)^{2 / \gamma} \leqslant n^{1-2 / \gamma} \sigma_{m}^{2}\right\} .
$$

Тогда при достаточно большом $n$ справедливо утверждение теоремы 14 и существует построение, для которого

$$
\mathrm{E}\left(\Delta_{n}(X, Y)\right)^{\gamma} \ll_{\psi, b, \gamma, \lambda} \max \left\{n^{(\gamma-\delta(\gamma-2)) / 2}\left(\log ^{*} n\right)^{\delta \gamma(\gamma+1) / 2}, n^{(\gamma-r(\gamma-2)) / 2}\right\},
$$

где

$$
r=\frac{b-1}{2 b-1+2 \psi}, \quad \delta=\frac{2(b-1)}{2 b+\gamma}, \quad \lambda=\mathrm{E}\|X\|^{\gamma} .
$$

ПримеР 4. Предположим, что в условиях примера 3 выполнено соотношение (79). Выберем

$$
d=\min \left\{m: n^{2 / \gamma} m^{2 \psi+1}<n m \sigma_{m}^{2}\right\} \geqslant 1
$$

Если условие (74) не выполнено, следует выбирать $d$ для применения теоремы 13 не по формуле (96), а с помощью соотношения

$$
d=\max \left\{m: C(\gamma) K^{2 / \gamma} m^{1+\gamma / 2}\left(\log ^{*} m\right)^{\gamma+1} \leqslant n^{1-2 / \gamma}\right\} .
$$

Тогда при достаточно большом $n$ справедливо утверждение теоремы 13 с оценкой

$$
\mathrm{E}\left(\Delta_{n}(X, Y)\right)^{\gamma} \ll_{\psi, b, \gamma, K} \max \left\{n^{(\gamma-\mu(\gamma-2)) / 2}\left(\log ^{*} n\right)^{\mu \gamma(\gamma+1) / 2}, n^{(\gamma-\rho(\gamma-2)) / 2}\right\} \mathrm{E}\|X\|^{\gamma},
$$

где

$$
\rho=\frac{b-1}{b+2 \psi}, \quad \mu=\frac{2(b-1)}{2+\gamma}
$$


ПримеР 5. Пусть $\sigma_{m}^{2}=1 /\left(m\left(\log ^{*} m\right)^{1+\tau}\right), m=1,2, \ldots$, где $\tau>0$. Через $\lfloor x\rfloor$ мы будем обозначать целую часть числа $x$. Выберем

$$
d=\left\lfloor n^{\varepsilon}\right\rfloor, \quad \text { где } \varepsilon=\frac{\gamma-2}{\gamma(\gamma+22)} .
$$

Тогда при достаточно большом $n$ выполнено условие (76) и справедливо утверждение теоремы 14 с оценкой

$$
\mathrm{E}\left(\Delta_{n}(X, Y)\right)^{\gamma} \ll_{\gamma, \tau}\left(\frac{n}{\left(\log ^{*} n\right)^{\tau}}\right)^{\gamma / 2} .
$$

Сравним оценки сверху, полученные в примерах 1, 3 и 5 с помощью теоремы 14, с оценкой снизу

$$
\mathrm{E}\left(\Delta_{n}(X, Y)\right)^{\gamma} \gg_{\gamma}\left(n B_{k}^{2}\right)^{\gamma / 2}
$$

вытекающей из (85).

В примере 1 оценка снизу (102) далека от оценки сверху (88). Нетрудно подсчитать, что в примере 3 натуральное число $k$, определенное равенством (84), удовлетворяет соотношению $k \asymp_{b, \lambda} n^{1 / b}, B_{k}^{2} \asymp_{b, \lambda} n^{(1-b) / b}$, а оценка снизу (102) имеет порядок $O\left(n^{\gamma / 2 b}\right)$. Это показывает, что порядок оценок сверху не случайно растет с ростом $\gamma$. Отметим, что при больших значениях $\gamma$ и $b$ порядок оценок сверху близок к $n^{\gamma / 4}$.

При относительно небольших значениях $\gamma$ и $b$ на порядок оценок существенно влияет величина $\psi$, которая возникает из-за достаточно больших степеней размерности в оценках погрешности в теоремах 11 и 12. Возможные уточнения теорем 11 и 12 должны привести к улучшению порядка точности аппроксимации как в примерах 1-4, так и в теоремах 13 и 14.

В примере 5 легко проверяется, что $k \asymp_{\tau} n /\left(\log ^{*} n\right)^{1+\tau}$. Тем самым, оценки снизу и сверху имеют одинаковый порядок $O\left(\left(n /\left(\log ^{*} n\right)^{\tau}\right)^{\gamma / 2}\right)$, так что теорема 14 дает правильный порядок точности аппроксимации. То же самое произойдет, если дисперсии $\sigma_{m}^{2}$ координат убывают еще медленнее, чем в примере 5. При этом порядок оценки может быть сделан сколь угодно близким к тривиальному порядку $O\left(n^{\gamma / 2}\right)$.

\section{1. Правильные по порядку оценки в бесконечномерном случае}

В этом разделе мы приведем результаты, опубликованные в работе автоpa [102] (теорема 17 и следствие 8). В них утверждается, что если последовательность собственных чисел $\sigma_{m}^{2}$ убывает медленно, то теоремы 13 и 14 дают оценки с правильным порядком точности аппроксимации. В теореме 17 и следствии 8 сформулированы условия, при которых порядки оценок сверху и снизу совпадают. Наличие оценок с правильным порядком свидетельствует о том, что метод построения, использованный при их получении, достаточно хорошо соответствует поставленной задаче. 
ТеОрема 17. Предположим, что $X$ есть Н-значный случайный вектор c $\mathrm{E} X=0 u \mathrm{E}\|X\|^{\gamma}<\infty$ при некотором $\gamma>2$. Пусть $F=\mathscr{L}(X) u$

$$
B_{d}^{2} \asymp_{F} \varphi(d+1), \quad d=0,1,2, \ldots,
$$

где невозрастающая функция $\varphi(x), x \geqslant 1$, удовлетворяет соотношению

$$
\varphi\left(x^{\lambda}\right) \asymp_{\lambda, F} \varphi(x) \quad \text { при всех } \lambda>1 .
$$

Предположим, что $\beta \geqslant 1$ u

$$
K_{\beta}=\sup _{1 \leqslant d<\infty} d^{-\gamma \beta / 2} \mathrm{E}\left\|\mathbb{D}_{d}^{-1 / 2} X_{(d)}\right\|^{\gamma}<\infty .
$$

Тогда существует такое построение, что

$$
\mathrm{E}\left(\Delta_{n}(X, Y)\right)^{\gamma} \ll_{\gamma, \beta, F}\left(n B_{n}^{2}\right)^{\gamma / 2} .
$$

C другой стороны, существует случайный вектор $X=\left(\mathscr{X}_{1}, \mathscr{X}_{2}, \ldots\right)$, принимающий значения в пространстве $\mathbf{H}$, удовлетворяющий соотношениям (64), (72) u $\mathrm{E}\|X\|^{\gamma}<\infty$ при всех $\gamma \geqslant 0$ и такой, что для любого построения справедлива оченка снизу

$$
\mathrm{E}\left(\Delta_{n}(X, Y)\right)^{\gamma} \gg_{\gamma}\left(n B_{n}^{2}\right)^{\gamma / 2}
$$

СледствиЕ 8. Утверждение теоремы 17 останется справедливым, если условие (105) заменить на условие

$$
\sigma_{d}^{2} \gg_{\beta, F} d^{-\beta}, \quad d=1,2, \ldots,
$$

при некотором $\beta \geqslant 1$.

Следствие 8 легко выводится из теоремы 17, поскольку из (78) и (108) следует выполнение условия (105). Однако условие (108) выражено в терминах собственных чисел $\sigma_{m}^{2}$, в то время как в условии (105) участвуют моменты векторов $\mathbb{D}_{d}^{-1 / 2} X_{(d)}$. Условие (105) можно рассматривать как естественное обобщение условия (79). Отметим, что следствие 8 можно вывести непосредственно из теорем 14 и 16. Выполнение условия (108) не следует из (103) и (104).

Правая часть неравенства (106) может не стремиться к нулю при $n \rightarrow \infty$. Чтобы удостовериться в том, что это неравенство дает содержательную оценку точности сильной аппроксимации в бесконечномерном принципе инвариантности, достаточно заметить, что неравенство (106) эквивалентно неравенству

$$
\mathrm{E}\left(\Delta_{n}\left(\frac{X}{\sqrt{n}}, \frac{Y}{\sqrt{n}}\right)\right)^{\gamma} \ll_{\gamma, \beta, F} B_{n}^{\gamma} \underset{n \rightarrow \infty}{\longrightarrow} 0 .
$$

С помощью теоремы П.5 из монографии [81; теорема П.5 из приложения] легко показать (см. доказательство теоремы 17 в [102]), что из (103) и (104) следует существование такого $\mu>0$, что

$$
B_{n}^{2} \gg_{F}\left(\log ^{*} n\right)^{-\mu}
$$


Так что, хотя неравенство (106) и дает правильные по порядку оценки в условиях теорем 17 и следствия 8, этот порядок оказывается не сильнее некоторой степени логарифма.

Анализ доказательства теорем 17 и следствия 8 показывает, что возможность получения правильных по порядку оценок основана на степенной зависимости констант от размерности в теоремах 13 и 14, которая в свою очередь связана со степенными оценками для констант, полученными в работах [41], [43], [95] и [96] (см. теоремы 2, 3, 11 и 12). Возможные уточнения зависимости констант от размерности в результатах работ [41], [43], [95] и [96] должны привести к улучшению порядка точности аппроксимации в теоремах 13 и 14 .

Заметим, что последнее утверждение теоремы 17 вытекает из теоремы 16 с $\lambda=B_{0}$. В качестве $\mathscr{X}_{m}$ (координат вектора $X$ ) при применении теоремы 16 используются независимые случайные величины, принимающие значения $-B_{0}$, 0 и $B_{0}$ с вероятностями

$$
\mathrm{P}\left\{\mathscr{X}_{m}= \pm B_{0}\right\}=\frac{\sigma_{m}^{2}}{2 B_{0}^{2}}, \quad \mathrm{P}\left\{\mathscr{X}_{m}=0\right\}=1-\frac{\sigma_{m}^{2}}{B_{0}^{2}}, \quad m=1,2, \ldots
$$

Условие (104) родственно условию медленного изменения функции $\varphi$ :

$$
\frac{\varphi(\lambda x)}{\varphi(x)} \underset{x \rightarrow \infty}{\longrightarrow} 1 \quad \text { при всех } \lambda>1,
$$

из которого следует, что

$$
x^{s} \varphi(x) \underset{x \rightarrow \infty}{\longrightarrow} \infty \quad \text { при всех } s>0
$$

(см. [81]). Заменяя условие (104) на условие (112) и пользуясь соотношением (113), можно доказать справедливость оценки

$$
\mathrm{E}\left(\Delta_{n}(X, Y)\right)^{\gamma} \ll_{\gamma, \beta, F}\left(n B_{d}^{2}\right)^{\gamma / 2}
$$

с $d$, определенным в (100). Последнее утверждение теоремы 17 по-прежнему

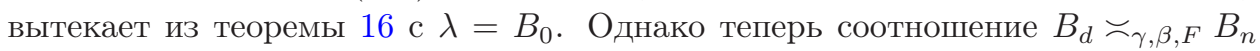
может не выполняться. Так будет, например, если функция $\varphi(x)$ задана равенством $\varphi(x)=\exp \left(-\left(\log ^{*} x\right)^{1 / 2}\right)$.

В качестве примера распределения, удовлетворяющего условиям следствия 8, можно взять распределение из примера 5. Приведем еще один пример.

ПримеР 6. Пусть $s$ - целое неотрицательное число и

$$
\sigma_{m}^{2}=\frac{1}{m \log ^{*} m \log ^{*} \log ^{*} m \ldots \underbrace{\log ^{*} \ldots \log ^{*}}_{s \text { раз }} m(\underbrace{\log ^{*} \ldots \log ^{*} m}_{s+1 \text { раз }})^{1+\tau}}
$$

при $m=1,2, \ldots$, где $\tau>0$. Тогда справедливо утверждение следствия 8 с оптимальной по порядку оценкой

$$
\mathrm{E}\left(\Delta_{n}(X, Y)\right)^{\gamma} \ll_{\gamma, \tau, s}(\underbrace{\left.\frac{n}{\left(\log ^{*} \ldots \log ^{*} n\right.} n\right)^{\tau}}_{s+1 \text { раз }})^{\gamma / 2} .
$$


Аналогичные примеры получаются, если дисперсии координат $\sigma_{m}^{2}$ убывают еще медленнее, чем в примере 2. При этом порядок оценки может быть сделан сколь угодно близким к тривиальному порядку $O\left(n^{\gamma / 2}\right)$. С помощью примера 2 можно построить большое количество аналогичных примеров, домножая $\sigma_{m}^{2}$ на логарифмические множители более высоких порядков или на не очень быстро растущие функции от этих множителей.

Автор благодарен А. И. Саханенко за чрезвычайно полезные и содержательные замечания, позволившие существенно улучшить изложение.

\section{Список литературы}

[1] P. Bártfai, "Die Bestimmung der zu einem wiederkehrenden Prozess gehörenden Verteilungfunktion aus den mit Fehlern behafteten Daten einer einzigen Realisation", Studia Sci. Math. Hungar., 1 (1966), 161-168.

[2] В. Бенткус, Ф. Гётце, В. Паулаускас, А. Рачкаускас, "Точность гауссовской аппроксимации в банаховых пространствах", Теория вероятностей-6. Предельные теоремы теории вероятностей, Итоги науки и техники. Соврем. проблемы. матем. Фундам. напр., 81, ВИНИТИ, М., 1991, 39-139; англ. пер.: V. Bentkus, F. Götze, V. Paulauskas, A. Račkauskas, "The accuracy of Gaussian approximation in Banach spaces", Limit theorems of probability theory, Springer-Verlag, Berlin, 2000, 25-111.

[3] E. Berger, Fast sichere Approximationen von Partialsummen unabhängiger und stationärer ergodischer Folgen von Zufallsvectoren, Dissertation, Universität zu Göttingen, 1982, 73 pp.

[4] I. Berkes, W. Philipp, "Approximation theorems for independent and weakly dependent random vectors", Ann. Probab., 7:1 (1979), 29-54.

[5] I. Berkes, S. Hörmann, "Almost sure invariance principles for dependent sequences", Electr. J. Probab., 2013 (to appear).

[6] I. Berkes, Weidong Liu, Wei Biao Wu, "Komlós-Major-Tusnády approximation under dependence", Ann. Probab., 2013 (to appear).

[7] П. Биллингсли, Сходимость вероятностных мер, Наука, М., 1977, 351 с.; пер. с англ.: P. Billingsley, Convergence of probability measures, John Wiley \& Sons, Inc., New York-London-Sydney, 1968, xii+253 pp.

[8] И.С. Борисов, "K вопросу о скорости сходимости в принципе инвариантности Донскера-Прохорова", Теория вероятн. и ее примен., 28:2 (1983), 367-372; англ. пер.: I.S. Borisov, "On the rate of convergence in Donsker-Prohorov invariance principle", Theory Probab. Appl., 28:2 (1984), 388-393.

[9] А.А. Боровков, "О скорости сходимости в принципе инвариантности", Теория вероятн. и ее примен., 18:2 (1973), 217-234; англ. пер.: А. A. Borovkov, "On the rate of convergence for the invariance principle", Theory Probab. Appl., 18:2 (1972), 207-225.

[10] А. А. Боровков, А. А. Могульский, А. И. Саханенко, "Предельные теоремы для случайных процессов", Теория вероятностей - 7, Итоги науки и техники. Соврем. пробл. матем. Фундам. напр., 82, ВИНИТИ, М., 1995, 5-194.

[11] А. А. Боровков, А. И. Саханенко, "Об оценках скорости сходимости в принципе инвариантности для банаховых пространств", Теория вероятн. и ее примен., 25:4 (1980), 734-744; англ. пер.: А. А. Borovkov, А. I. Sahanenko, "On estimates of the rate of convergence in the invariance principle for Banach spaces", Theory Probab. Appl., 25:4 (1981), 721-731. 
[12] A. A. Borovkov, A. I. Sakhanenko, "On the rate of convergence in invariance principle", Probability theory and mathematical statistics (Tbilisi, 1982), Lecture Notes in Math., 1021, Springer, Berlin, 1983, 59-66.

[13] К. А. Боровков, "О скорости сходимости в принципе инвариантности для гильбертова пространства”, Теория вероятн. и ее примен., 29:3 (1984), 532-535; англ. пер.: К. A. Borovkov, "On the convergence rate in the invariance principle", Theory Probab. Appl., 29:3 (1985), 550-553.

[14] А.Н. Бородин, И. А. Ибрагимов, "Предельные теоремы для функционалов от случайных блужданий", Тр. МИАН СССР, 195, Наука, СПб., 1994, 3-285; англ. пер.: A. N. Borodin, I. A. Ibragimov, "Limit theorems for functionals of random walks", Proc. Steklov Inst. Math., 195:2 (1995), 1-259.

[15] A. Bovier, D. M. Mason, "Extreme value behaviour in the Hopfield model", Ann. Appl. Probab., 11:1 (2001), 91-120.

[16] L. Breiman, "On the tail behavior of sums of independent random variables", Z. Wahrscheinlichkeitstheor. verw. Geb., 9:1 (1967), 20-25.

[17] S. Chatterjee, "A new approach to strong embeddings", Probab. Theory Related Fields, 152:1-2 (2012), 231-264.

[18] M. Csörgo", "A glimpse of the KMT (1975) approximation of empirical processes by Brownian bridges via quantiles", Acta Sci. Math. (Szeged), 73:1-2 (2007), 349-366.

[19] S. Csörgo", P. Hall, "The Komlós-Major-Tusnády approximations and their applications", Austral. J. Statist., 26:2 (1984), 189-218.

[20] M. Csörgö, P. Révész, "A new method to prove Strassen type laws of invariance principle. I", Z. Wahrscheinlichkeitstheor. verw. Geb., 31 (1974/1975), 255-259; "II", 261-269.

[21] M. Csörgö, P. Révész, Strong approximations in probability and statistics, Probab. Math. Statist., Academic Press, Inc., New York-London, 1981, 284 pp.

[22] A. de Acosta, "Inequalities for $B$-valued random vectors with applications to the strong law of large numbers", Ann. Probab., 9:1 (1981), 157-161.

[23] V. H. de la Peña, E. Giné, Decoupling. From dependence to independence. Randomly stopped processes. U-statistics and processes. Martingales and beyond, Probab. Appl. (N. Y.), Springer-Verlag, New York, 1999, xvi+392 pp.

[24] M. D. Donsker, An invariance principle for certain probability limit theorems, Mem. Amer. Math. Soc., 1951, № 6, 1951, 12 pp.

[25] M.D. Donsker, "Justification and extension of Doob's heuristic approach to the Kolmogorov-Smirnov theorems", Ann. Math. Statist., 23:2 (1952), 277-281.

[26] J. L. Doob, "Heuristic approach to the Kolmogorov-Smirnov theorems", Ann. Math. Statist., 20:3 (1949), 393-403.

[27] R. M. Dudley, "Distances of probability measures and random variables", Ann. Math. Statist., 39:5 (1968), 1563-1572.

[28] U. Einmahl, A refinement of the KMT inequality for partial sum strong approximation, Techn. Rep. Ser. Lab. Res. Statist. Probab., 88, Carleton Univ., Ottawa, ON, 1986, 113 pp.

[29] U. Einmahl, "A useful estimate in the multidimensional invariance principle", Probab. Theory Related Fields, 76:1 (1987), 81-101.

[30] U. Einmahl, "Strong invariance principles for partial sums of independent random vectors", Ann. Probab., 15:4 (1987), 1419-1440.

[31] U. Einmahl, "Extensions of results of Komlós, Major, and Tusnády to the multivariate case", J. Multivariate Anal., 28:1 (1989), 20-68.

[32] U. Einmahl, "A new strong invariance principle for sums of independent random vectors", Вероятность и статистика. 14-2, Зап. научн. сем. ПОМИ, 364, ПОМИ, СПб., 2009, 5-31; J. Math. Sci. (N. Y.), 163:4 (2010), 311-327. 
[33] U. Einmahl, J. Kuelbs, "Cluster sets for a generalized law of iterated logarithm in Banach spaces", Ann. Probab., 29:4 (2001), 1451-1475.

[34] U. Einmahl, D. M. Mason, "Rates of clustering in Strassen's LIL for partial sums processes", Probab. Theory Related Fields, 97:4 (1993), 479-487.

[35] U. Einmahl, D. M. Mason, "Gaussian approximation of local empirical processes indexed by functions", Probab. Theory Related Fields, 107:3 (1997), 283-311.

[36] P. Erdös, M. Kac, "On certain limit theorems of the theory of probability", Bull. Amer. Math. Soc., 52:4 (1946), 292-302.

[37] N. Etemadi, "On some classical results in probability theory", Sankhyā Ser. A, 47:2 (1985), 215-221.

[38] Д.Х. Фук, С. В. Нагаев, "Вероятностные неравенства для сумм независимых случайных величин", Теория вероятн. и ее примен., 16:4 (1971), 660-675; англ. пер.: D. H. Fuk, S. V. Nagaev, "Probability inequalities for sums of independent random variables", Theory Probab. Appl., 16:4 (1971), 643-660.

[39] B. Gentz, M. Löwe, "Fluctuations in the Hopfield model at the critical temperature", Markov Process. Related Fields, 5:4 (1999), 423-449.

[40] F. Götze, A. Yu. Zaitsev, "Multidimensional Hungarian construction for vectors with almost Gaussian smooth distributions", Asymptotic methods in probability and statistics with applications (St. Petersburg, 1998), Stat. Ind. Technol., eds. N. Balakrishnan, I. Ibragimov, V. Nevzorov, Birkhäuser, Boston, MA, 2001, 101-132.

[41] F. Götze, A. Yu. Zaitsev, "Bounds for the rate of strong approximation in the multidimensional invariance principle", Теория вероятн. и ее примен., 53:1 (2008), 100-123; Theory Probab. Appl., 53:1 (2009), 59-80.

[42] Ф. Гётце, А. Ю. Зайцев, “Точность аппроксимации в многомерном принципе инвариантности для сумм независимых одинаково распределенных случайных векторов с конечными моментами", Вероятность и статистика. 15, Зап. научн. сем. ПОМИ, 368, ПОМИ, СПб., 2009, 110-121; англ. пер.: F. Götze, A. Yu. Zaitsev, "Rates of approximation in the multidimensional invariance principle for sums of i.i.d. random vectors with finite moments", J. Math. Sci. (N. Y.), 167:4 (2010), 495-500.

[43] Ф. Гётце, А. Ю. Зайцев, "Оценки точности сильной аппроксимации в гильбертовом пространстве", Сиб. матем. журн., 52:4 (2011), 796-808; англ. пер.: F. Götze, A. Yu. Zaitsev, "Estimates for the rate of strong approximation in Hilbert space", Sib. Math. J., 52:4 (2011), 628-638.

[44] В.В. Городецкий, "О скорости сходимости в многомерном принципе инвариантности", Теория вероятн. и ее примен., 20:3 (1975), 642-649; англ. пер.: V. V. Gorodetskii, "On the rate of convergence for the multidimensional invariance principle", Theory Probab. Appl., 20:3 (1976), 631-638.

[45] I. Grama, M. Nussbaum, "A functional Hungarian construction for sums of independent random variables", Ann. Inst. H. Poincaré Probab. Statist., 38:6 (2002), 923-957.

[46] N. C. Jain, K. Jogdeo, W. F. Stout, "Upper and lower functions for martingales and mixing processes", Ann. Probab., 3:1 (1975), 119-145.

[47] W. B. Johnson, G. Schechtman, J. Zinn, "Best constants in moment inequalities for linear combinations of independent and exchangeable random variables", Ann. Probab., 13:1 (1985), 234-253.

[48] J. Kiefer, "On the deviations in the Skorokhod-Strassen approximation scheme", Z. Wahrscheinlichkeitstheor. verw. Geb., 13:3-4 (1969), 321-332.

[49] V. Koltchinskii, "Komlos-Major-Tusnády approximation for the general empirical process and Haar expansions of classes of functions", J. Theoret. Probab., 7:1 (1994), $73-118$. 
[50] J. Komlós, P. Major, G. Tusnády, "An approximation of partial sums of independent RV's, and the sample DF. I", Z. Wahrscheinlichkeitstheor. verw. Geb., 32:1-2 (1975), 111-131; "II", 34:1 (1976), 33-58.

[51] S. Kwapień, W. Woyczyński, Random series and stochastic integrals: single and multiple, Probab. Appl., Birkhäuser Boston, Inc., Boston, MA, 1992, xvi+360 pp.

[52] М. А. Лифшиц, Лекиии по сильной аппроксимачии, Изд-во СПбГУ, СПб., 2007, $32 \mathrm{c}$.

[53] P. Major, "The approximation of partial sums of independent RV's", Z. Wahrscheinlichkeitstheor. verw. Geb., 35:3 (1976), 213-220.

[54] P. Major, "Approximation of partial sums of i.i.d. r.v.s when summands have only two moments", Z. Wahrscheinlichkeitstheor. verw. Geb., 35:3 (1976), 221-229.

[55] P. Major, "On the invariance principle for sums of independent identically distributed random variables", J. Multivariate Anal., 8:4 (1978), 487-517.

[56] P. Major, "An improvement of Strassen's invariance principle", Ann. Probab., 7:1 (1979), 55-61.

[57] P. Massart, "Strong approximation for multivariate empirical and related processes, via KMT constructions", Ann. Probab., 17:1 (1989), 266-291.

[58] S. J. Montgomery-Smith, "Comparison of sums of independent identically distributed random vectors", Probab. Math. Statist., 14:2 (1993), 281-285.

[59] S. V. Nagaev, "Large deviations of sums of independent random variables", Ann. Probab., 7:5 (1979), 745-789.

[60] В. В. Петров, Предельные теоремы для сумм независимых случайных величин, Наука, М., 1987, 318 с.

[61] W. Philipp, "Almost sure invariance principles for sums of $B$-valued random variables", Probability in Banach spaces II, Proceedings of the Second International Conference (Oberwolfach, 1978), Lecture Notes in Math., 709, Springer, Berlin, 1979, 171-193.

[62] Ю.В. Прохоров, "Сходимость случайных процессов и предельные теоремы теории вероятностей", Теория вероятн. и ее примен., 1 (1956), 177-238.

[63] Qi-Man Shao, "On a problem of Csörgo” and Révész", Ann. Probab., 17:2 (1989), 809-812.

[64] Qi Man Shao, "Strong approximation theorems for independent random variables and their applications", J. Multivariate Anal., 52:1 (1995), 107-130.

[65] E. Rio, "Strong approximation for set-indexed partial sum processes via K.M.T. constructions. I", Ann. Probab., 21:2 (1993), 759-790; "II", 21:3 (1993), 1706-1727.

[66] E. Rio, "Local invariance principles and their applications to density estimation", Probab. Theory Related Fields, 98:1 (1994), 21-45.

[67] E. Rio, "Vitesses de convergence dans le principe d'invariance faible pour la fonction de répartition empirique multivariée", C. R. Acad. Sci. Paris Sér. I Math., 322:2 (1996), 169-172.

[68] M. Rosenblatt, "Remarks on a multivariate transformation", Ann. Math. Statist., 23:1 (1952), 470-472.

[69] H. P. Rosenthal, "On the subspaces of $L^{p}(p>2)$ spanned by sequences of independent random variables", Israel J. Math., 8:3 (1970), 273-303.

[70] H. P. Rosenthal, "On the span in $L^{p}$ of sequences of independent random variables. II", Proceedings of the Sixth Berkeley Symposium on Mathematical Statistics and Probability, v. II: Probability theory (Univ. California, Berkeley, CA, 1970/1971), Univ. California Press, Berkeley, CA, 1972, 149-167.

[71] Р. Рудзкис, "О вероятностях больших уклонений случайных векторов", Литов. матем. сб., 23:1 (1983), 195-204; англ. пер.: R. Rudzkis, "Probabilities of large deviations of random vectors", Lith. Math. J., 23:1 (1983), 113-120. 
[72] А. И. Саханенко, "Скорость сходимости в принципе инвариантности для разнораспределенных величин с экспоненциальными моментами", Предельные теоремы для сумм случайных величин, Тр. Ин-та матем. СО АН СССР, 3, Наука, Новосибирск, 1984, 4-49.

[73] А.И. Саханенко, "Оценки в принципе инвариантности", Пределъные теоремъ теории вероятностей, Тр. Ин-та матем. СО АН СССР, 5, Наука, Новосибирск, 1985, 27-44.

[74] A. I. Sakhanenko, "Simple method of obtaining estimates in the invariance principle", Probability theory and mathematical statistics (Kyoto, 1986), Lecture Notes in Math., 1299, Springer, Berlin, 1988, 430-443.

[75] А. И. Саханенко, "О точности сильной нормальной аппроксимации в принципе инвариантности”, Асимптотический анализ распределений случайных прочессов, Тр. Ин-та матем. СО АН СССР, 13, Наука, Новосибирск, 1989, 40-66.

[76] A. I. Sakhanenko, "A new way to obtain estimates in the invariance principle", High dimensional probability II (Seattle, WA, 1999), Progr. Probab., 47, Birkhäuser, Boston, MA, 2000, 223-245.

[77] А.И. Саханенко, "Оценки в принципе инвариантности в терминах срезанных степенных моментов", Сиб. матем. журн., 47:6 (2006), 1355-1371; англ. пер.: A. I. Sakhanenko, "Estimates in the invariance principle in terms of truncated power moments", Sib. Math. J., 47:6 (2006), 1113-1127.

[78] А. И. Саханенко, "Одна общая оценка в принципе инвариантности", Сиб. матем. журн., 52:4 (2011), 876-893; англ. пер.: A. I. Sakhanenko, "A general estimate in the invariance principle", Siberian Math. J., 52:4 (2011), 696-710.

[79] Л. Саулис, "О больших уклонениях для случайных векторов для некоторых классов множеств. І", Литов. матем. сб., 23:3 (1983), 142-154; англ. пер.: L. Saulis, "Large deviations for random vectors for certain classes of sets. I", Lith. Math. J., 23:3 (1983), 308-317.

[80] G. Schay, "Nearest random variables with given distributions", Ann. Probab., 2:1 (1974), 163-166.

[81] Е. Сенета, Правильно меняющиеся функиии, Наука, М., 1985, 142 с.; пер. с англ.: E. Seneta, Regularly varying functions, Lecture Notes in Math., 508, Springer-Verlag, Berlin-New York, 1976, v+112 pp.

[82] А. В. Скороход, Исследования по теории случайных прочессов, Изд-во Киевского ун-та, Киев, 1961, 216 с.; англ. пер.: А. V. Skorokhod, Studies in the theory of random processes, Addison-Wesley, Reading, MA, 1965, viii+199 pp.

[83] V.A. Statulevičius, "On large deviations", Z. Wahrscheinlichkeitstheor. verw. Geb., 6:2 (1966), 133-144.

[84] V. Strassen, "An invariance principle for the law of iterated logarithm", Z. Wahrscheinlichkeitstheor. verw. Geb., 3 (1964), 211-226.

[85] V. Strassen, "The existence of probability measures with given marginals", Ann. Math. Statist., 36:2 (1965), 423-439.

[86] V. Strassen, "Almost sure behavior of sums of independent random variables and martingales", Proceedings of the Fifth Berkeley Symposium on Mathematical Statistics and Probability, v. II: Contributions to probability theory, Part 1 (Berkeley, CA, 1965/66), Univ. California Press, Berkeley, CA, 1967, 315-343.

[87] А.Ю. Зайцев, "Об аппроксимации гауссовскими распределениями при выполнении многомерных аналогов условий Бернштейна", Докл. AH CCCP, 276:5 (1984), 1046-1048; англ. пер.: A. Yu. Zaitsev, "Approximation by Gaussian distributions with satisfaction of multidimensional analogues of Bernstein's conditions", Soviet Math. Dokl., 29:3 (1984), 624-626. 
[88] А.Ю. Зайцев, О гауссовской аппроксимации сверток при выполнении многомерных аналогов условий неравенства Бернштейна, Препринт Р-9-84, ЛОМИ, Л., 1984, 54 c.

[89] А. Ю. Зайцев, "Оценки расстояния Леви-Прохорова в многомерной центральной предельной теореме для случайных векторов с конечными экспоненциальными моментами", Теория вероятн. и ее примен., 31:2 (1986), 246-265; англ. пер.: A. Yu. Zaitsev, "Estimates of the Lévy-Prohorov distance in the multivariate central limit theorem for random vectors with finite exponential moments", Theory Probab. Appl., 31:2 (1986), 203-220.

[90] A. Yu. Zaitsev, "On the Gaussian approximation of convolutions under multidimensional analogues of S. N. Bernstein's inequality conditions", Probab. Theory Related Fields, 74:4 (1987), 535-566.

[91] А. Ю. Зайцев, "О связи между двумя классами вероятностных распределений", Кольиа и модули. Пределъные теоремы теории вероятностей, Вып. 2, Изд-во ЛГУ, Л., 1988, 153-158.

[92] А. Ю. Зайцев, "Многомерный вариант второй равномерной предельной теоремы Колмогорова", Теория вероятн. и ее примен., 34:1 (1989), 128-151; англ. пер.: A. Yu. Zaitsev, "Multidimensional version of the second uniform limit theorem of Kolmogorov", Theory Probab. Appl., 34:1 (1989), 108-128.

[93] А. Ю. Зайцев, “Оценки квантилей гладких условных распределений и многомерный принцип инвариантности", Сиб. матем. журн., 37:4 (1996), 807-831; англ. пер.: A. Yu. Zaitsev, "Estimates for the quantiles of smooth conditional distributions and the multidimensional invariance principle", Sib. Math. J., 37:4 (1996), 706-729.

[94] А. Ю. Зайцев, "Многомерный вариант результата Комлоша, Майора и Тушнади для векторов с конечными экспоненциальными моментами", Докл. РАH, 357:6 (1997), 731-733; англ. пер.: A. Yu. Zajtsev, "Multidimensional variant of the Komlós, Major and Tusnády results for vectors with finite exponential moments", Dokl. Math., 56:3 (1997), 935-937.

[95] A. Yu. Zaitsev, "Multidimensional version of the results of Komlós, Major, and Tusnády for vectors with finite exponential moments", ESAIM Probab. Statist., 2 (1998), 41-108 (electronic).

[96] A. Yu. Zaitsev, "Multidimensional version of a results of Sakhanenko in the invariance principle for vectors with finite exponential moments. I", Теория вероятн. и ее примен., 45:4 (2000), 718-738; Theory Probab. Appl., 45:4 (2001), 624-641; "II", Теория вероятн. и ее примен., 46:3 (2001), 535-561; Theory Probab. Appl., 46:3 (2002), 490-514; "III", Теория вероятн. и ее примен., 46:4 (2001), 744-769; Тhеоry Probab. Appl., 46:4 (2002), 676-698.

[97] A. Yu. Zaitsev, "On the strong Gaussian approximation in multidimensional case", Ann. I.S.U.P., 45:2-3 (2001), 3-7.

[98] A. Yu. Zaitsev, "Estimates for the strong approximation in multidimensional central limit theorem", Proceedings of the International Congress of Mathematicians, v. III (Beijing, 2002), Higher Education Press, Bejing, 2002, 107-116.

[99] А. Ю. Зайцев, "Оценки точности сильной аппроксимации в многомерном принципе инвариантности", Вероятность и статистика. 10, Зап. научн. сем. ПОМИ, 339, ПОМИ, СПб., 2006, 37-53; англ. пер.: А. Yu. Zaitsev, "Estimates for the rate of strong approximation in the multidimensional invariance principle", J. Math. Sci. (N. Y.), 145:2 (2007), 4856-4865.

[100] А. Ю. Зайцев, "Оценки точности сильной гауссовской аппроксимации сумм независимых одинаково распределенных случайных векторов", Вероятность и статистика. 12, Зап. научн. сем. ПОМИ, 351, ПОМИ, СПб., 2007, 141-157; англ. пер.: A. Yu. Zaitsev, "Estimates for the rate of strong Gaussian approximation for sums of i.i.d. multidimensional random vectors", J. Math. Sci. (N. Y.), 152:6 (2008), 875-884. 
[101] А. Ю. Зайцев, “Точность сильной гауссовской аппроксимации для сумм независимых одинаково распределенных случайных векторов", Вероятность и статистика. 14-2, Зап. научн. сем. ПОМИ, 364, ПОМИ, СПб., 2009, 148-165; англ. пер.: A. Yu. Zaitsev, "The rate of Gaussian strong approximation for the sums of i.i.d. multidimensional random vectors", J. Math. Sci. (N. Y.), 163:4 (2009), 399-408.

[102] А. Ю. Зайцев, "Оптимальные оценки точности сильной аппроксимации в бесконечномерном принципе инвариантности”, Вероятность и статистика. 17, Зап. научн. сем. ПОМИ, 396, ПОМИ, СПб., 2011, 93-101; англ. пер.: А. Yu. Zaitsev, "Optimal estimates for the rate of strong Gaussian approximation in a Hilbert space", J. Math. Sci. (N. Y.), 188:6 (2013), 689-693.

А. Ю. Зайцев (А. Yu. Zaitsev)

Санкт-Петербургское отделение

Математического института им. В. А. Стеклова РАН;

Санкт-Петербургский государственный университет

E-mail: zaitsev@pdmi.ras.ru
Поступила в редакцию

09.04.2013 